\title{
The steady propagation of a surfactant-laden liquid plug in a two-dimensional channel
}

\author{
Hideki Fujioka and James B. Grotberg ${ }^{a)}$ \\ Department of Biomedical Engineering, The University of Michigan, Ann Arbor, Michigan 48109
}

(Received 4 November 2004; accepted 18 May 2005; published online 22 July 2005)

\begin{abstract}
In this study, we investigate the steady propagation of a liquid plug in a two-dimensional channel lined by a uniform, thin liquid film. The liquid contains soluble surfactant that can exist both in the bulk fluid and on the air-liquid interface. The Navier-Stokes equations with free-surface boundary conditions and the surfactant transport equations are solved using a finite volume numerical scheme. The adsorption/desorption process of the surfactant is modeled based on pulmonary surfactant properties. As the plug propagates, the front meniscus sweeps preexisting interfacial surfactant from the precursor film, and the surfactant accumulates on the front meniscus interface. As the front meniscus converges on the precursor film from the region where the interfacial surfactant concentration is maximized, the Marangoni stress opposes the flow. In this region, the Marangoni stress results in nearly zero surface velocity, which causes the precursor film thickness near the meniscus to be thicker than the leading film thickness. Since the peaks of wall pressure and wall shear stress occur due to narrowing of the film thickness, the observed increase of the minimum film thickness weakens these stresses. In the thicker film region, however, the drag forces increase due to an increase in the surfactant concentration. This causes the overall pressure drop across the plug to increase as a result of the increasing surfactant concentration. A recirculation flow forms inside the plug core and is skewed toward the rear meniscus as the Reynolds number increases. When no surfactant exists, the recirculation flow is in contact with both the front and the rear interfaces. As the surfactant concentration increases, the Marangoni stress begins to rigidify the front interface and forces the recirculation flow away from the front interface. Subsequently, the recirculation flow is directed away from the rear interface in a manner similar to that for the front interface. When the plug length is shorter, this change in recirculation pattern occurs at a smaller surfactant concentration. (C) 2005 American Institute of Physics. [DOI: 10.1063/1.1948907]
\end{abstract}

\section{INTRODUCTION}

Pulmonary surfactant plays a vital role in reducing the surface tension of the liquid lining alveoli and airways. The surfactant is necessary to reduce the work required to expand the lungs with each breath. Prematurely born neonates can develop respiratory distress syndrome (RDS) because their lungs are not sufficiently mature to produce adequate quantities of the surfactant. RDS is characterized by low lung compliance, areas of atelectasis, airway closure, poor arterial oxygenation, and fluid-filled alveoli. ${ }^{1}$ A thin liquid layer coating the inside of airways can be unstable and forms a meniscus or plug due to a capillary instability. ${ }^{2-4}$ When lungsurfactant availability is reduced, this tendency can be increased. ${ }^{5}$ This will occur particularly near the end of expiration in distal airways, causing the airways to be closed due to the plug formation. The lung volume at which this airway closure begins is called the closing volume of the lung, which can be measured through pulmonary function tests. Once formed, the liquid plug will then be convected by the inhaled air, and it can eventually rupture under certain conditions or persist under others. During this airway-reopening process, pulmonary epithelial cells may be damaged by me-

\footnotetext{
a) Author to whom correspondence should be addressed. Electronic mail: grotberg@umich.edu
}

chanical stresses associated with the fluid motion ${ }^{6}$ and the plug rupture.

Surfactant replacement therapy (SRT) is now a standard treatment for premature neonates suffering from RDS and has a dramatic impact on infant survival rate. ${ }^{7-10}$ The most common protocol for instilling surfactant is injecting four quarters dose aliquots into the trachea, although variations of this procedure have been used clinically. ${ }^{11}$ Liquid is also instilled into the pulmonary airways in partial liquid ventilation (PLV) (Refs. 12-18 and drug delivery. ${ }^{19-28}$ In these medical treatments, the formation of a liquid plug in the trachea before inspiration is important in creating a uniform liquid distribution throughout the lung. ${ }^{29}$ As the plug propagates during inspiration, it deposits a trailing liquid film on the airway wall and may eventually rupture. The trailing film thickness is an important factor for the liquid delivery. The film thickness increases with the plug propagation speed.

Most theoretical studies on plug propagation in liquidlined channels and tubes, with or without surfactant, have been carried out in the Stokes flow regime, neglecting the effects of fluid inertia, that is, in the limit of zero Reynolds number. Waters and Grotberg ${ }^{30}$ asymptotically investigated the effects of soluble surfactant on liquid plug propagation. They assumed that the bulk surfactant concentration and the fluid pressure in the plug core were constant. They showed 
that the driving pressure difference $\Delta P$ for a given capillary number, $\mathrm{Ca}=\mu^{*} U^{*} / \sigma^{*}$, increases with increasing surface elasticity, but decreases with precursor film thickness. Here, $\mu^{*}$ is the fluid viscosity, $\sigma^{*}$ is the surface tension, and $U^{*}$ is the fluid speed. The trailing film thickness increases with $\Delta P$, but at a slower rate when the surface elasticity is larger. Howell et al. ${ }^{31}$ analyzed surfactant-free liquid plug propagation in a prewetted flexible tube in the asymptotic limit $\mathrm{Ca}$ $\rightarrow 0$. They identified a critically imposed pressure drop above, which the liquid plug will eventually rupture. Fujioka and Grotberg $^{32}$ numerically investigated the effect of the plug length and the fluid inertia on a liquid plug with a surfactant-free interface. The results showed that the trailing film thickness decreases as the plug length decreases below the channel width, and for sufficiently short plugs, there is a significant interaction between the leading and the trailing menisci and their local flow effects.

When the plug length is long enough, the interaction between both menisci can be negligible, ${ }^{32}$ therefore, analysis for the plug propagation can be reduced to a long bubble propagation. Ginley and Radke ${ }^{33}$ assumed that the surfactant concentration in the bulk fluid was uniform and that the adsorption/desorption process was the controlling mechanism for the surfactant transport. Their results indicate that, under these conditions, the surface concentration of surfactant increases in the thin-film region compared to the bubble front so that the film thickness decreases compared with the surfactant-free case. Ratulowski and $\mathrm{Chang}^{34}$ carried out a more comprehensive asymptotic analysis for different convective, diffusive, and kinetic timescales at low bubble speeds. They showed that if the transport in the film is mass transfer limited so that a bulk surfactant concentration gradient exists in the film, the film thickness increases by a maximum factor of $4^{2 / 3}$. There are some numerical studies focused on the effect of surfactant on a semi-infinite bubble. ${ }^{35-37}$ Ghadiali and Gaver $^{36}$ numerically investigated the effect of a soluble surfactant on the fluid dynamics of a semi-infinite bubble propagating in a capillary tube filled with a viscous liquid. They found that the surfactant properties could strongly influence the interfacial pressure drop through modification of the surface tension and the creation of the Marangoni stress. They showed when the bulk Péclet number is large or sorption rates are low, bulk surfactant concentration profiles can substantially differ from nearequilibrium approximations and can result in film thinning.

Bilek et al. ${ }^{6}$ investigated surface-tension-induced lung epithelial cell damage in a model of airway reopening, consisting of a semi-infinite bubble propagating in a narrow fluid-filled channel lined with pulmonary epithelial cells. They showed that cell damage increased with decreasing reopening velocity, and that the presence of pulmonary surfactant prevented this injury. Using a computational model, they demonstrated that the cells lining the channel walls could experience different types of mechanical stress as the finger of air propagated over these cells: shear stress, pressure, shear stress gradient, and pressure gradient. They concluded that the steep pressure gradient near the finger front was the most likely cause of the observed cellular damage. Our previous study ${ }^{32}$ of a finite-length liquid plug is comparable to

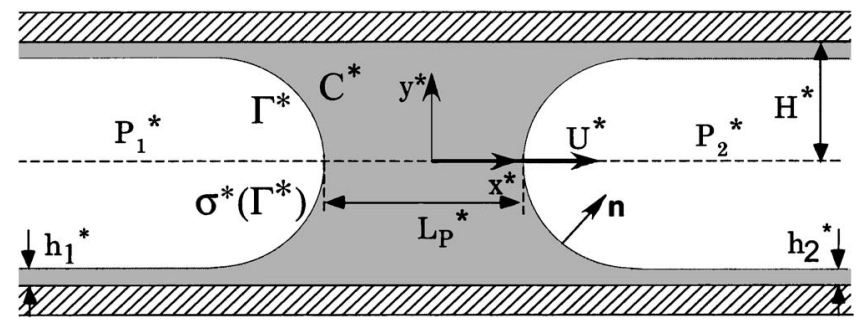

FIG. 1. A sketch of the liquid plug model. A pressure difference between the front and the rear air fingers, $\Delta P^{*}=P_{1}^{*}-P_{2}^{*}$, drives the liquid plug of the length $L_{P}$ with constant speed $U^{*}$ in a two-dimensional channel of halfwidth $H^{*}$ lined by a precursor film of thickness $h_{2}^{*}$. For steady state, $h_{2}^{*}$ is equal to the trailing film thickness at the rear end boundary $h_{1}^{*}$. The liquid contains surfactant molecules, which can exist either in the bulk solution with concentration $C^{*}$ or adsorbed onto the interface with concentration $\Gamma^{*}$. The surface tension $\sigma^{*}$ is a function of $\Gamma^{*}$.

that of Bilek et al. ${ }^{6}$ in the limit of long plug length and zero Reynolds number by focusing on the rear interface. However, we also showed that these mechanical stresses have a sharp peak where the precursor liquid film thickness is the smallest, which is in the capillary wave located at the front interface. Particularly, with a finite Reynolds number, these stresses and their variations are significantly larger than those at the rear, and consequently larger than those examined by Bilek et al. ${ }^{6}$ In addition, the single finger of air has a trailing film, which increases in thickness as $\mathrm{Ca}$ increases for zero Reynolds number, so shear stresses decrease with increasing $\mathrm{Ca}$. For the plug at finite Reynolds number, both the front and the rear shear stresses and pressure amplitudes increase as the velocity increases, so there are qualitative and quantitative differences between the two models. These facts suggest that there is a higher risk of pulmonary epithelial cell damage in the front of the liquid plug than in the rear.

The capillary wave is developed due to a force balance between the surface tension and the fluid forces. When surfactant exists on the interface, it affects the surface tension and creates a gradient in the surface tension that induces the Marangoni stress. The Marangoni stress affects local flow and changes stresses acting on the wall. In this paper, we numerically investigate the propagation of a liquid plug, which is laden with soluble surfactant, varying plug propagation speed, plug length, and the concentration of surfactant. The present study simulates the surfactant transport phenomena with the regime for pulmonary surfactant, which is characterized by surfactant adsorption rate onto the interface that is faster than desorption rate into the bulk, and convective transport that is much faster than the adsorption and the desorption processes.

\section{METHOD}

\section{A. Model description}

In this paper, we investigate the steady propagation of a liquid plug of length $L_{P}^{*}$ inside a two-dimensional channel of width $2 H^{*}$ as shown in Fig. 1. A pressure difference between the front and the rear air fingers, $\Delta P^{*}=P_{1}^{*}-P_{2}^{*}$, drives the liquid plug consisting of an incompressible, Newtonian fluid with a constant speed $U^{*}$. The gas-phase viscosity is assumed 
negligible so that only fluid dynamics of the liquid phase is considered. The liquid contains surfactant molecules, which can exist either in the bulk solution with concentration $C^{*}$ or adsorbed onto the interface with concentration $\Gamma^{*}$. At the precursor film far ahead of the plug, the bulk surfactant concentration is prescribed to be $C_{0}^{*}$. The interfacial surfactant concentration there is in equilibrium with $C_{0}^{*}$. In this computational analysis, the plug propagation speed $U^{*}$ is specified, so that $\Delta P^{*}$ is one of the unknown quantities. The plug is assumed symmetrical about the channel centerline, and only the lower half of the domain is considered. Under steadystate conditions, the precursor film thickness $h_{2}^{*}$ has to be equal to the trailing film thickness $h_{1}^{*}$. In a moving frame with a constant velocity $U^{*}$, the flow inside the liquid plug is described by the nondimensional Navier-Stokes equations and the continuity equation,

$$
\begin{aligned}
& \operatorname{Re}(\mathbf{u} \cdot \boldsymbol{\nabla}) \mathbf{u}=-\boldsymbol{\nabla} p+\nabla^{2} \mathbf{u}, \\
& \boldsymbol{\nabla} \cdot \mathbf{u}=0,
\end{aligned}
$$

and the surfactant mass balance in the bulk,

$$
\operatorname{Pe} \boldsymbol{\nabla} \cdot(\mathbf{u} C)=\nabla^{2} C,
$$

where $\mathbf{u}$ is the fluid velocity scaled on $U^{*}, \mathbf{u}=\mathbf{u}^{*} / U^{*}, p$ is the fluid pressure scaled on the viscous pressure scale, $p$ $=p^{*} /\left(\mu^{*} U^{*} / H^{*}\right), \quad C$ is the bulk surfactant concentration scaled with $C_{\mathrm{cmc}}^{*}$, which is the critical micelle bulk surfactant concentration; the bulk surfactant concentration cannot exceed this value without forming micelles, $(x, y)$ are the Cartesian coordinates scaled on $H^{*}$, and $\boldsymbol{\nabla}=(\partial / \partial x, \partial / \partial y)$. The Reynolds number, which represents the ratio of inertia to viscous forces, is defined as $\operatorname{Re}=\rho^{*} U^{*} H^{*} / \mu^{*}$, where $\rho^{*}$ is the liquid density. The bulk Péclet number, which represents the ratio of convection to diffusion, is defined as $\mathrm{Pe}=U^{*} H^{*} / D^{*}$, where $D^{*}$ is the diffusion coefficient of the surfactant in the liquid phase. A frame of reference is chosen so that $y= \pm 1$ denote the channel walls, $y=0$ represents the centerline of the channel, and $x=0$ denotes the middle section of the plug. The no-slip condition and no penetration of the surfactant on the channel walls require

$$
\mathbf{u}=(-1,0), \quad \frac{\partial C}{\partial y}=0 \quad \text { at } y=-1 .
$$

Symmetry conditions are applied at the channel center, $y=0$. The boundary conditions at the end boundary in the precursor film are the following: the pressure is uniform in $y$ and equal to the front gas pressure, $P_{2}=0$; the fully developed condition is applied for the velocity; and the bulk surfactant concentration is prescribed as $C=C_{0}$. At the end boundary in the trailing film the pressure is uniform in $y$ and equal to the rear gas pressure $P_{1}$, which is a part of the solution, and the fully developed condition is applied for the velocity, $\partial C / \partial x=0$, for the bulk surfactant concentration.

At each air-liquid interface, we apply a kinematic boundary condition

$$
\mathbf{u} \cdot \mathbf{n}=0,
$$

where $\mathbf{n}$ is the unit vector normal to the interface. A balance of normal forces which accounts for the jump in stress due to surface tension is

$$
-p \mathbf{n}+\left(\boldsymbol{\nabla} \mathbf{u}+\boldsymbol{\nabla} \mathbf{u}^{T}\right) \cdot \mathbf{n}=\mathrm{Ca}^{-1}\left(\kappa \sigma \mathbf{n}+\nabla_{s} \sigma\right)-P_{a} \mathbf{n},
$$

where $P_{a}$ represents the air pressure ( $a=1$ or 2$), \kappa=\kappa^{*} H^{*}$ is the dimensionless interface curvature, $\nabla_{s}=(\mathbf{I}-\mathbf{n n}) \cdot \boldsymbol{\nabla}, \sigma$ $=\sigma^{*} / \sigma_{M}^{*}$ is the dimensionless surface tension, $\sigma_{M}^{*}$ is surface tension when there is no surfactant exists in the interface. $\mathrm{Ca}=\mu^{*} U^{*} / \sigma_{M}^{*}$ is the capillary number that relates viscous force to surface tension. The transport equation for the interfacial surfactant is

$$
\mathrm{Pe}_{s} \boldsymbol{\nabla}_{s} \cdot\left(\mathbf{u}_{s} \Gamma\right)-\nabla_{s}^{2} \Gamma=j_{n},
$$

where $\mathbf{u}_{s}$ is tangential component of the fluid velocity on the surface, $\mathrm{Pe}_{s}=U^{*} H^{*} / D_{s}^{*}$ is the surface Péclet number, $D_{s}^{*}$ is the diffusion coefficient of the surfactant in the air-liquid interface, and $\Gamma=\Gamma^{*} / \Gamma_{\infty}^{*}$, where $\Gamma_{\infty}^{*}$ is the maximum equilibrium concentration; the interfacial surfactant concentration cannot exceed this value without dynamic compression of the interface. ${ }^{38} j_{n}$ is the surfactant flux from the bulk to the interface. The boundary condition at both meniscus tips is $\partial \Gamma / \partial s=0$ because of symmetry. $\Gamma$ is uniform for $s$ far away from the plug, so that $\partial \Gamma / \partial s=0$ is applied at both ends of the film. The boundary condition for the bulk surfactant concentration along the interface is that the diffusion of the bulk surfactant balances with the surfactant flux from the bulk to the interface, $j_{n}$, so that

$$
-\frac{1}{\chi} \frac{\mathrm{Pe}_{s}}{\mathrm{Pe}}(\mathbf{n} \cdot \nabla) C=j_{n},
$$

where $\chi=\Gamma_{\infty}^{*} / C_{\mathrm{cmc}}^{*} H^{*}$ is the dimensionless adsorption depth which is a parameter of the critical micelle bulk surfactant concentration.

In a static system, as the bulk concentration increases, the interface becomes saturated with surfactant and $\Gamma^{*}$ attains $\Gamma_{\infty}^{*}$. In a dynamic system, $\Gamma^{*}$ can exceed $\Gamma_{\infty}^{*}$ in the region where the surfactant accumulates due to the surface flow. The surface surfactant monolayer will buckle or collapse when $\Gamma^{*}$ exceeds the maximum dynamic surface concentration $\Gamma_{\max }^{*} \cdot{ }^{38,39}$ The interfacial surfactant directly modifies the surface tension which decreases as $\Gamma^{*}$ increases. The surface tension $\sigma^{*}$ is almost a linear function of $\Gamma^{*}$ in an equilibrium state for $\Gamma^{*}<\Gamma_{\infty}^{*}$. For $\Gamma_{\infty}^{*} \leqslant \Gamma^{*}<\Gamma_{\max }^{*}$, the $\sigma^{*}-\Gamma^{*}$ curve behaves nonlinearly, and $\sigma^{*}$ decays to a constant value when $\Gamma^{*}$ reaches $\Gamma_{\text {max }}^{*}$ because the surface tension is determined by only the surfactant layer in contact with the air phase. ${ }^{38,39}$ In this study, the relation between the surface tension and the interfacial surfactant concentration is approximated as the flowing equation: 


$$
\sigma= \begin{cases}1-E \Gamma & (\Gamma<1), \\ (1-E) \exp \left[\frac{E}{1-E}(1-\Gamma)\right] & (\Gamma \geqslant 1),\end{cases}
$$

where $E=-\left.\left(\Gamma_{\infty}^{*} / \sigma_{M}^{*}\right)\left(\partial \sigma^{*} / \partial \Gamma^{*}\right)\right|_{\Gamma^{*}<\Gamma_{\infty}^{*}}$ is the elasticity number, which represents the ability of the surfactant to modify the surface tension.

The surfactant flux from the bulk into the interface involves an adsorption and a desorption process from the subsurface to the interface in the bulk. In this study, we employed Langmuir-Hinshelwood kinetics ${ }^{36,40}$ to determine the form of $j_{n}$ when the interfacial surfactant concentration is smaller than the maximum equilibrium concentration $\Gamma_{\infty}^{*}$; but when the surfactant concentration exceeds the maximum equilibrium concentration, the adsorption is switched off:

$$
j_{n}= \begin{cases}K_{a} C_{s}(1-\Gamma)-K_{d} \Gamma & (\Gamma<1), \\ -K_{d} \Gamma & (\Gamma \geqslant 1),\end{cases}
$$

where $K_{a}=k_{a}^{*} C_{\mathrm{cmc}}^{*} H^{* 2} / D_{s}^{*}$ represents the ratio of adsorption to surface diffusion rate and $K_{d}=k_{d}^{*} H^{* 2} / D_{s_{*}^{*}}^{*}$ is the ratio of desorption to surface diffusion rate, $k_{a}^{*}$ and $k_{d}^{*}$ are adsorption and desorption rates, respectively, and $C_{s}$ is the dimensionless bulk surfactant concentration in the subsurface.

\section{B. Numerical methods}

The calculation starts from an assumed velocity profile and interface shape. The grid inside the domain is generated and then the coordinate transform-matrices are calculated. The governing equation (1) and (2) and for the flow and the interface shape, subject to the boundary conditions described above, are solved numerically using the SIMPLER algorithm $^{41}$ with the boundary fitted coordinate transformation. ${ }^{42}$ A kinematic update scheme ${ }^{43}$ is employed to correct the interface shape. The details are given in our previous paper. ${ }^{32}$ The surfactant transport equations (3) and (7) and are solved with the finite volume method and incorporated with the SIMPLER algorithm. At both ends of the thin-film regions, the velocity is uniform in $y$, which is $\mathbf{u}$ $=(-1,0)$. The flow rate coming into the plug, $-h_{2}$, has to match with the flow rate going out from the plug, $-h_{1}$, because of the steady state. This yields $h_{2}=h_{1}$. Since the trailing film thickness $h_{1}$ is one of the unknown quantities, the precursor film thickness $h_{2}$ has to be specified as $h_{2}=h_{1}$ at each iteration step. These steps described above are repeated until all the computing variables converge.

The grid nodes were generated using the elliptic generation system, ${ }^{44}$ where a uniform computational domain $(\xi, \eta)$ is mapped to inside a plug domain $(x, y)$ that is determined by solution of two-dimensional Poisson's equations. The right-hand side of Poisson's equation, which controls the grid space, was defined to gather the grid lines to the free surface. The node points, which are located inside from the free surface, are adjusted to make a local orthogonal grid at the free surface. ${ }^{45}$ To check the grid convergence, we compared the trailing film thickness and the pressure drop across the plug in three different mesh systems: (a) 5504 total node points -9 node points in film depth, 52 node points between the front and the rear menisci tips, and 272 node points on each free surface; (b) 6304 total node points -9 node points in film depth, 77 node points between the front and the rear menisci tips, and 272 node points on each free surface; and (c) 8704 total node points-14 node points in film depth, 77 node points between the front and the rear menisci tips, and 272 node points on each free surface. The difference of the trailing film thickness was within $2 \%$, and the difference of the pressure drop was within $1 \%$ in three mesh systems, when the dimensionless parameters were $L_{P}=2, \mathrm{Ca}=0.05$, $\mathrm{Re}=50, \mathrm{Pe}=500, \mathrm{Pe}_{s}=5000, K_{a}=10^{4}, K_{d}=10^{2}, \chi=10^{-2}, E$ $=0.7$, and $C_{0}=10^{-4}$, where $C_{0}$ is the bulk surfactant concentration prescribed in the leading front edge of the film. So in this study, the mesh system (b) is employed for $L_{P}=2$. For $L_{P}=1$ and $0.5,52$, and 32 node points between the front and the rear menisci tips are employed. We compared the trailing film thickness with studies for a semi-infinite bubble propagation without surfactant; our previous paper ${ }^{32}$ showed that our result in the Stokes flow regime agrees with the numerical studies by Giavedoni and Saita ${ }^{46}$ and Halpern and Gaver. ${ }^{47}$ For small $\mathrm{Ca}$, the trailing film thickness approaches the theoretical result by Bretherton. ${ }^{48}$ Also our result in finite Re regime agrees with other numerical studies. ${ }^{46,49}$

\section{RESULTS}

The dynamics of liquid plug propagation can vary significantly, depending on the breathing rate, the tidal volume, the airway radius, the properties of the instilled liquid, and the properties and the amounts of surfactant. Since Re and $\mathrm{Ca}$ are both functions of the plug speed $U^{*}$, it is convenient to introduce the parameter, $\lambda=\operatorname{Re} / \mathrm{Ca}=\rho^{*} \sigma_{M}^{*} H^{*} / \mu^{* 2}$. When $U^{*}$ changes, Re and Ca vary, but $\lambda=\mathrm{Re} / \mathrm{Ca}$ remains constant; it depends on the geometry of the channel and fluid properties of the plug. Since the properties of Survanta are $\rho^{*}$ $=0.94 \mathrm{~g} / \mathrm{cm}^{3}, \mu^{*}=42 \mathrm{cP}$, and $\sigma_{M}^{*}=25$ dynes $/ \mathrm{cm}^{50},{ }^{50}$ these dimensionless parameters for the SRT are in the range of 1 $<\lambda<20,0.1<\operatorname{Re}<40$, and $0.1<\mathrm{Ca}<2$ for airway generations from 0 to 13 in an infant's lung. Therefore, at higher generations, the Reynolds number is quite small. In the PLV or the airway closure, the values of $\operatorname{Re}$ and $\lambda$ are somewhat larger than in the SRT, and the inertial effects cannot be neglected. For example, for perfluorocarborn Peflubron (Alliance Pharmaceuticals, San Diego, CA) $\rho^{*}=1.93 \mathrm{~g} / \mathrm{cm}^{3}$, $\mu^{*}=2.1 \mathrm{cP}$, and $\sigma_{M}^{*}=18$ dynes $/ \mathrm{cm}$. ${ }^{50}$ Thus, the range of $\lambda$ is $\approx 10^{3}<\lambda<10^{4}$ and of $\operatorname{Re}$ is $10<\operatorname{Re}<1000$.

The Péclet number is also a function of $U^{*}$. In this study, the Schmidt number, which is the ratio of kinematic viscosity to diffusivity (bulk or surface), is used to determine the Péclet number as a function of $\mathrm{Re} ; \mathrm{Pe}_{s}=\mathrm{Sc}_{s} \mathrm{Re}$ is the Péclet number for the interfacial surfactant and $\mathrm{Pe}=\mathrm{Sc} \mathrm{Re}$ is the Péclet number of the bulk surfactant.

The dimensionless parameters for the surfactant are defined referring to the adsorption and the desorption kinetics of pulmonary surfactant, which are $k_{a}^{*} \sim(1.7$ $\left.\times 10^{3}\right) \mathrm{cm}^{3} \mathrm{~g}^{-1} \mathrm{~s}^{-1}$ and $k_{d}^{*} \sim\left(1.7 \times 10^{-2}\right) \mathrm{s}^{-1} \cdot{ }^{39,51}$ Assuming the critical micelle bulk concentration for pulmonary surfactant is $C_{\mathrm{cmc}}^{*} \sim 10^{-3} \mathrm{~g} \mathrm{~cm}^{-3},{ }^{52}$ the ratio of the dimensionless adsorption and desorption rates, $K_{d} / K_{a}=k_{d}^{*} / k_{a}^{*} C_{\mathrm{cmc}}^{*}$, is $\approx 10^{-2}$, which means the desorption is much slower than the 
adsorption. The maximum equilibrium interfacial concentration for the pulmonary surfactant is $\Gamma_{\infty}^{*} \sim(3.1$ $\left.\times 10^{-7}\right) \mathrm{g} \mathrm{cm}^{-2}{ }^{53}$ Assuming the channel half-width $H^{*}$ is of order of $10^{-1} \mathrm{~cm}$, the dimensionless adsorption depth is $\approx \chi=10^{-2}$. The dimensionless elasticity number is $\approx E=0.7 .^{39}$ Agrawal and Neuman ${ }^{54}$ measured the surface diffusivity $D_{s}^{*}$ of pulmonary surfactant in myristic acid and found $10^{-7}<D_{s}^{*}<\left(7 \times 10^{-5}\right) \mathrm{cm}^{2} \mathrm{~s}^{-1}$. In this study, $D_{s}^{*}$ $=10^{-6} \mathrm{~cm}^{2} \mathrm{~s}^{-1}$ is used to determine the dimensionless adsorption and desorption rates, $K_{a}=10^{4}$ and $K_{d}=10^{2}$.

The surface Péclet number $\mathrm{Pe}_{s}$ should be very large because the surface diffusivity is very small. For large $\mathrm{Pe}_{s}$, the surface convection dominates the transport and the adsorption/desorption process has relatively negligible effect. In this regime, the surfactant can be thought of as insoluble in the bulk. During the propagation of the plug, the front meniscus sweeps up preexisting surfactant and the surfactant accumulates; the surface surfactant concentration $\Gamma^{*}$ increases to a very large value. When $\Gamma^{*}$ exceeds the maximum dynamic surface concentration $\Gamma_{\max }^{*}$, the surface surfactant monolayer buckles or collapses and forms micelles. ${ }^{38,39,55-61}$ The micelles detach from the interface and transport in the bulk without desorption, which is not considered in the present transport model. Due to this limitation, the simulation has to be performed within the regime where micelles are not created. To keep the bulk surfactant concentration smaller than the critical micelle concentration and to keep the interfacial surfactant concentration smaller than the maximum dynamic surface concentration, we employed an even larger surface diffusivity; the Schmidt number for the interface, $\mathrm{Sc}_{s}=100$, is used to determine the surface Péclet number.

The diffusivity for pulmonary surfactant molecules in the bulk solution is $D^{*}=\left(5.4 \times 10^{-8}\right) \mathrm{cm}^{2} \mathrm{~s}^{-1}{ }^{51}$ Again, we employed even larger values of the bulk diffusivity for the transport system to be consistent; the Schmidt number for the bulk, $\mathrm{Sc}=10$, is used to determine the bulk Péclet number. However, in the present study, for $10 \leqslant \operatorname{Re} \leqslant 70$, the surfactant transport process is calculated with the bulk Péclet number in the range $100 \leqslant \mathrm{Pe} \leqslant 700$ and the surface Péclet number in the range $1000 \leqslant \mathrm{Pe}_{s} \leqslant 7000$, which is large enough to resolve the feature of the regime that the convective transport dominates diffusion.

In this paper, we employed the following values of the dimensionless parameters: $\lambda=1000, \mathrm{Sc}=10, \mathrm{Sc}_{s}=100, K_{a}$ $=10^{4}, K_{d}=10^{2}, \chi=10^{-2}, E=0.7$. In Sec. III A, we investigate the propagating liquid plug with $10 \leqslant \operatorname{Re} \leqslant 70$ for three different plug lengths, $L_{P}=0.5,1$, and 2 , when the bulk surfactant concentration prescribed at the end boundary of the precursor film is $C_{0}=10^{-4}$. The effect of $C_{0}$, in the range 0 $\leqslant C_{0} \leqslant 10^{-3}$, on the plug for $\operatorname{Re}=50, L_{P}=0.5,1$, and 2 is given in Sec. III B.

\section{A. The effect of the plug propagation speed $U^{\star}$}

In this section, we investigate the liquid plug behavior with changing propagation speed $U^{*}$ through the Reynolds number Re. The remaining dimensionless parameters are as above.

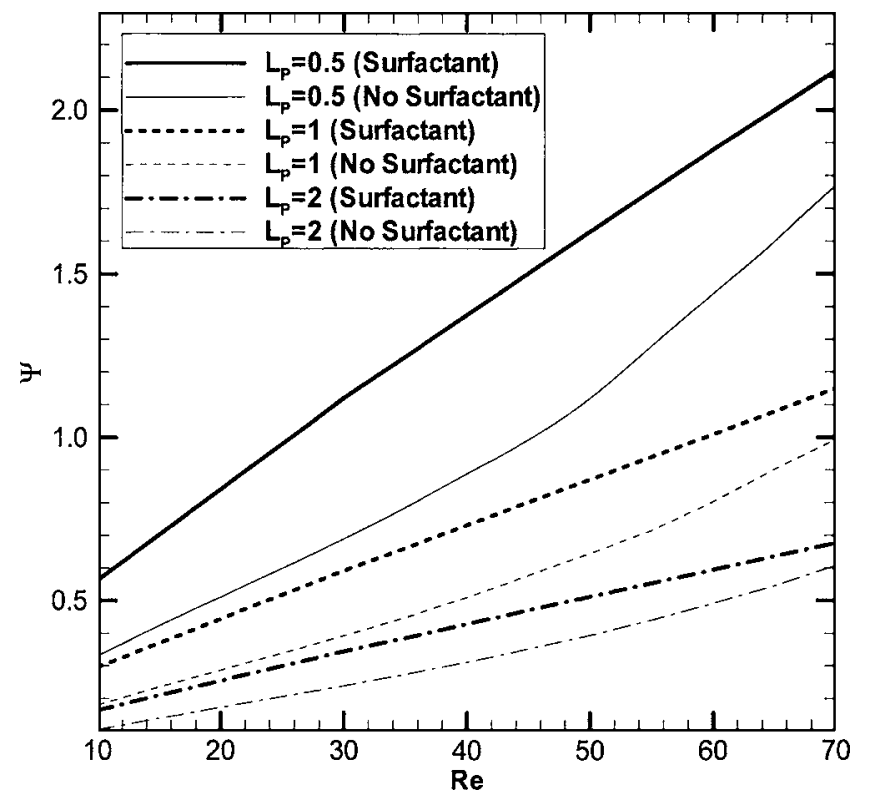

FIG. 2. The overall pressure drop across the plug divided by the plug length, $\Psi=\left(\Delta P^{*} / L_{P}^{*}\right)\left(H^{* 2} / \sigma_{M}^{*}\right)$, vs Re for $L_{P}=0.5,1$, and 2 . Thick lines represent $\Psi$ for the liquid plug with surfactant when $\lambda=1000, \mathrm{Sc}=10, \mathrm{Sc}_{s}=100, K_{a}$ $=10^{4}, K_{d}=10^{2}, \chi=10^{-2}, E=0.7$, and $C_{0}=10^{-4}$. Thin lines denoted with "No Surfactant" are the plug with surfactant-free interface when $\lambda=1000$.

Figure 2 shows the overall pressure drop across the plug divided by the plug length, $\Psi=\left(\Delta P^{*} / L_{P}^{*}\right)\left(H^{2} / \sigma_{M}^{*}\right)$ versus Re for three different plug lengths, $L_{P}=0.5,1$, and 2, with or without surfactant. The three thick lines (solid, dash, dash dot) represent $\Psi$ for the surfactant-laden liquid plug when the bulk surfactant concentration of $C_{0}=10^{-4}$ is prescribed at the precursor film far ahead of the plug. The three thin lines denote the "no surfactant" case. As Re increases, $\Psi$ increases monotonically for all cases. Comparing the cases with and without surfactant at a fixed $L_{P}, \Psi$ with surfactant is larger than $\Psi$ without surfactant for whole range of Re in Fig. 2; the surfactant increases the overall pressure drop across the plug. The curves of $\Psi$ with surfactant increase almost linearly with $\mathrm{Re}$, but the curves of $\Psi$ without surfactant increase like a quadratic function of Re, which has been discussed in our previous study $^{32}$ for the plug propagation without surfactant: approximating to $\Psi \sim \psi_{1} \operatorname{Re}+\psi_{2} \operatorname{Re}^{2}$, $\psi_{1}$ Re term comes from viscous dissipation in the plug core and $\psi_{2} \operatorname{Re}^{2}$ term comes from dynamic pressure that depends on the flow in the front and the rear transition regions. As $L_{P} \rightarrow 0$, the value of $\psi_{2}$ becomes larger; the dynamic pressure in the transition region dominates the pressure drop. As $L_{P}$ increases, because the dynamic pressure drop in the transition region becomes relatively smaller than the viscous dissipation in the plug core, $\Psi$ approaches the Poiseuille flow value, which is a linear function of $\operatorname{Re}$ as $\psi_{1} \rightarrow 3\left(1-h_{1}\right) / \lambda$ and $\psi_{2} \rightarrow 0$. The Marangoni stress induced by the surface tension gradient makes the interface less mobile, requiring a larger force to move the menisci. This situation is similar to the study by Park ${ }^{62}$ who showed that the pressure drop for a bubble in a liquid-filled channel increases as it propagates in a soluble surfactant solution. Since we assumed $\sigma$ to be a linear function of $\Gamma$ when $\Gamma<1$ as described in Eq. (9), the 


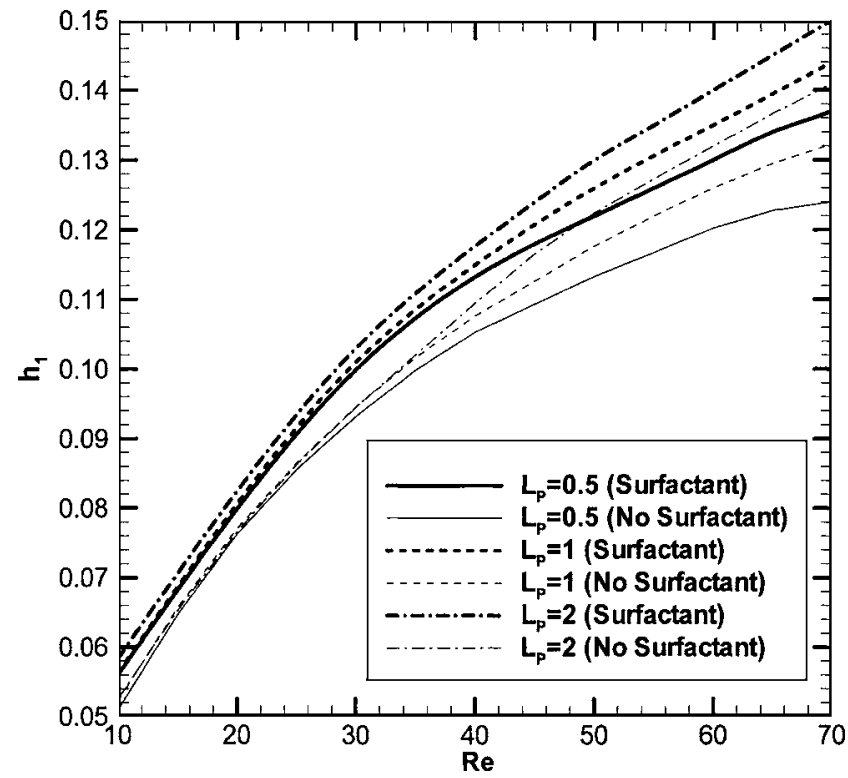

FIG. 3. The trailing film thickness $h_{1}$ as a function of Re for $L_{P}=0.5,1$, and 2. Thick lines represent $h_{1}$ for the liquid plug with surfactant when $\lambda$ $=1000, \mathrm{Sc}=10, \mathrm{Sc}_{s}=100, K_{a}=10^{4}, K_{d}=10^{2}, \chi=10^{-2}, E=0.7$, and $C_{0}=10^{-4}$. Thin lines denoted with no surfactant are the plug with surfactant-free interface when $\lambda=1000$.

Marangoni stress is a linear function of the surface concentration gradient that increases as the surface Péclet number increases, so that $\Psi$ increases linearly with the surfactant.

The trailing film thickness $h_{1}$ as a function of Re and $L_{P}$ is shown in Fig. 3. For all cases, $h_{1}$ increases as Re increases, which is rather attributed by the increase of $\mathrm{Ca}$ with $U^{*}$, because the effect of the fluid inertia on the trailing film thickness is moderate. ${ }^{46,49}$ Our previous study without the surfactant $^{32}$ showed that the interaction between the front and the rear menisci makes $h_{1}$ smaller and the changes in $h_{1}$ by $L_{P}$ increase with $\mathrm{Re}$. As $L_{P}$ decreases, the interaction between the front and the rear menisci makes the pressure between the menisci almost uniform. This results in smaller $h_{1}$. As $L_{P}$ decreases, $h_{1}$ with surfactant decreases in a manner similar to that for no-surfactant case. The curves of $h_{1}$ versus Re with surfactant are larger than without the surfactant at a fixed $L_{P}$; the surfactant thickens the trailing film thickness in a way similar to that for semi-infinite bubble propagation. ${ }^{37}$ The interfacial surfactant in the rear meniscus lowers the surface tension. An effective capillary number is larger than $\mathrm{Ca}$, which is defined by the surface tension in surfactant-free interface. Since $h_{1}$ increases with the capillary number, $h_{1}$ increases with the surfactant concentration. Also because the Marangoni stress acts from the meniscus tip toward the trailing film (it is shown later in Figs. 7 and 8), more liquid is induced to flow toward the trailing film, resulting in thicker $h_{1}$.

Figure 4, lower half, shows streamlines (black), isopressure lines (white), and the pressure field as grayscale inside the liquid plug for $\operatorname{Re}=50, \lambda=1000$ without surfactant, and $L_{P}=0.5$. Figure 4 , upper half, shows velocity vectors through the cross section at several values of $x$. Figures 5 and 6 also show isoconcentration lines (white) and the concentration field in grayscale for $L_{P}=0.5$ and $L_{P}=2$ with surfactant. At the far right of the domain, $x=23$ (not shown), the bulk surfactant concentration is set to $C=C_{0}=10^{-4}$ for the cases in Figs. 5 and 6 , the liquid pressure equals the gas pressure $P_{2}$, which we defined as the reference pressure and set to zero. At the far left of the domain, $x=-42$ (not shown), the boundary condition for the bulk surfactant is $\partial C / \partial x=0$ for the cases in Figs. 5 and 6; the pressure equals the gas pressure $P_{1}$, which is a part of the solution.

For the streamlines, there are two major flow regions in the domain. One is a band of streamlines passing through the plug from the precursor film to the trailing film. Another is a recirculation region inside the plug core that does not communicate directly with the precursor or the trailing films. At steady state, all the recirculation streamlines are closed and there is no convective momentum flux between these two regions. The center of the recirculation skews toward the rear meniscus due to the inertial effect. ${ }^{32}$ If there is no surfactant, Fig. 4 shows stagnation points on the front and the rear menisci at the tips (midline), as well as off the midline, a total of four in the half domain, all on the interface, i.e., there are six interfacial stagnation points in the whole domain. The streamline connecting these two points separates the recirculation and the band of streamlines regions. When the front and the rear menisci are close enough, the fluid between both the menisci is almost static; for $L_{P}=0.5$, Figs. 4 and 5, the recirculation velocity between the menisci is very small. Figure 5, now with soluble surfactant, shows for $L_{P}=0.5$ that in the rear meniscus there is just one interfacial stagnation point at the tip. The second stagnation point now appears inside the plug, on the midline, off the meniscus tip, close to the rear surface stagnation point. Similarly, in the front meniscus, there is one stagnation point on the interface at the meniscus tip. Another stagnation point appears inside the plug on the midline, off the meniscus tip. The recirculation zone is

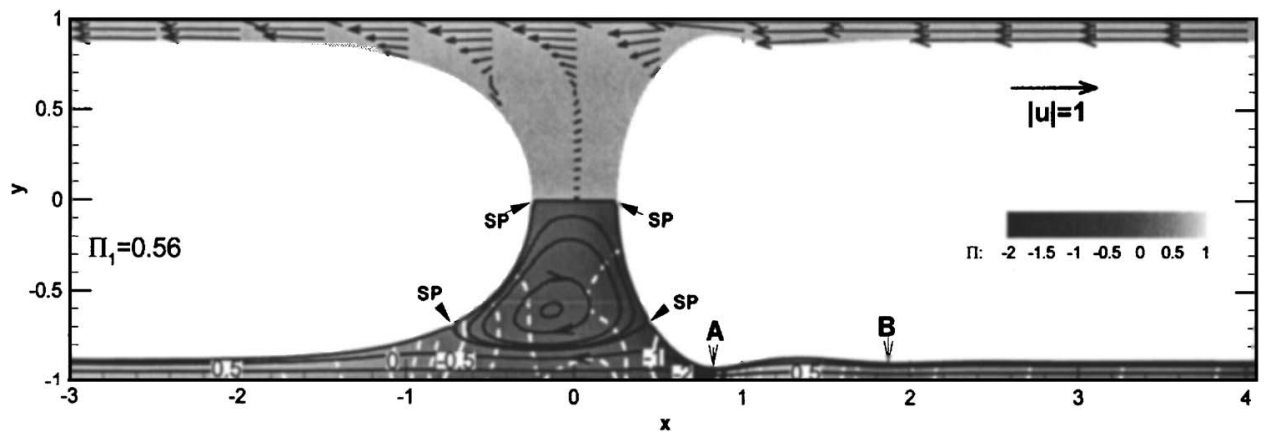

FIG. 4. Streamlines, velocity, pressure, and bulk surfactant concentration inside the liquid plug without surfactant for $L_{P}=0.5, \lambda=1000$, and $\mathrm{Re}=50$. 


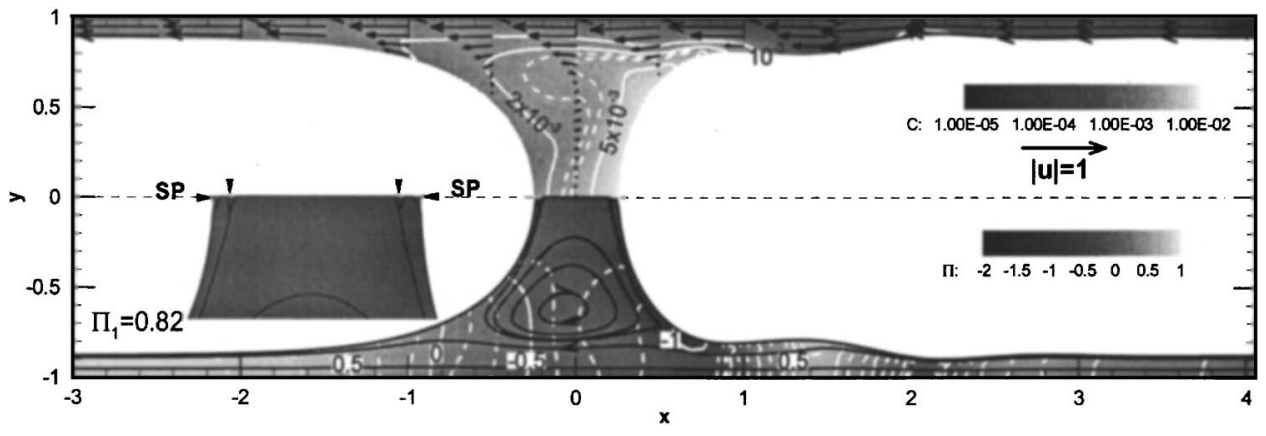

FIG. 5. Streamlines, velocity, pressure, and bulk surfactant concentration inside the liquid plug with surfactant for $L_{P}=0.5, \lambda=1000, \operatorname{Re}=50, \mathrm{Sc}=10$, $\mathrm{Sc}_{s}=100, K_{a}=10^{4}, K_{d}=10^{2}, \chi=10^{-2}$, $E=0.7$, and $C_{0}=10^{-4}$. no longer in contact with the front and the rear interfaces. There are four stagnation points in the whole domain, all on the midline.

For $L_{P}=2$ in Fig. 6, the front meniscus and its stagnation points are similar to the case of Fig. 5, one on the midline at the interface and one on the midline just interior to the interface. On the rear meniscus, however, the situation is like that in Fig. 4, where both stagnation points are on the interface, one at the midline and one off it. This streamline pattern varies with the surfactant concentration. The Marangoni stress causes the surface velocity to be almost zero and the recirculation zone to be off the interface, which is shown by Ghadiali and Gaver ${ }^{36}$ in their study for semi-infinite bubble propagation in a soluble surfactant solution. The details are shown in Sec. III B. We confirmed that the location of these stagnation points is not sensitive to the mesh systems (a), (b), and (c), described in Sec. II B.

The velocity distributions are plotted in the upper half of the plug picture in Fig. $4-L_{P}=0.5$ without surfactant, Fig. $5-L_{P}=0.5$, and Fig. $6-L_{P}=2$ with surfactant. The axial velocity at the wall is $u=-1$ in the reference frame. Our previous study for the plug propagation without surfactant shows when the front and the rear menisci are close enough; the fluid between both menisci is almost static. For Fig. 5, $L_{P}$ $=0.5$ with surfactant, because the plug is short, the velocity near the midline is fairly small; the axial velocity at $x=0$, $y=0$ is $u=0.003$. For Fig. $6, L_{P}=2$; now the plug is long enough for the flow to develop. The velocity profiles at the middle cross section, $x=0$, show a nearly parabolic shape and the axial velocity component at $x=0, y=0$ reaches $u$ $=0.23$.

The pressure field in the lower half of each plug picture in Figs. $4-6$ is $\Pi=\mathrm{Ca} p=p^{*} H / \sigma_{M}^{*}$ defined as the dimensional pressure scaled with the surface tension. Figure $4, L_{P}=0.5$ without surfactant, shows that $\Pi$ increases with a steep gra- dient along the rear meniscus as the rear meniscus converges on the trailing film, $-1.5<x<-0.6$. The pressure inside the plug core is smaller than the gas pressure on both sides due to the resultant effect of the surface tension. In the front meniscus, $\Pi$ ranges from -2.97 at the narrowest region of the precursor film to 0.62 . For Fig. $5, L_{P}=0.5$ with surfactant, at far left end in the trailing film, $\Pi_{1}$ is larger than in Fig. 4. In the rear transition region, $\Pi$ increases with a steep gradient along the rear meniscus as the rear meniscus converges on the trailing film, similar to the case without surfactant. In the front meniscus, the pressure shows a local minimum at $x=0.64$ in the transition region where the front meniscus converges on the precursor film. The film thickness shows a local minimum at $x=0.82$ (denoted by $\mathrm{A}$ ). When there is no surfactant acting on the interface, as Fig. 4 shows, the film thickness attains the smallest value at this location in the front transition region. For the surfactant case, Fig. 5 shows that the precursor film near the front meniscus, 0.8 $<x<1.6$, is thicker than the film thickness far ahead of the plug, and the minimum film thickness is observed where this thicker film region connects to the leading front precursor film (denoted by B). For Fig. 6, $L_{P}=2$ with surfactant, the pressure in the front and the rear transition regions shows profiles similar to the case in Fig. 5, but not in the plug core. There is a region where concentric isopressure lines appear due to centrifugal forces, ${ }^{32,49}$ the center of which is located at $x=-0.002, y=-0.599$ where $\Pi=-0.62$, while for Figs. 4 and 5 , $\Pi$ does not show such a region within the plug core, $-0.5<x<0.5$. This local minimum in $\Pi$ of Fig. 6 is located off the recirculation center because the pressure field here is a combination of the pressure gradient through the plug due to the viscous dissipation with the centrifugal pressure gradient across streamlines. The film thickness near the front meniscus, $1.6<x<2.4$ in Fig. 6 , is thicker than the precursor film thickness, and the minimum film thickness is observed

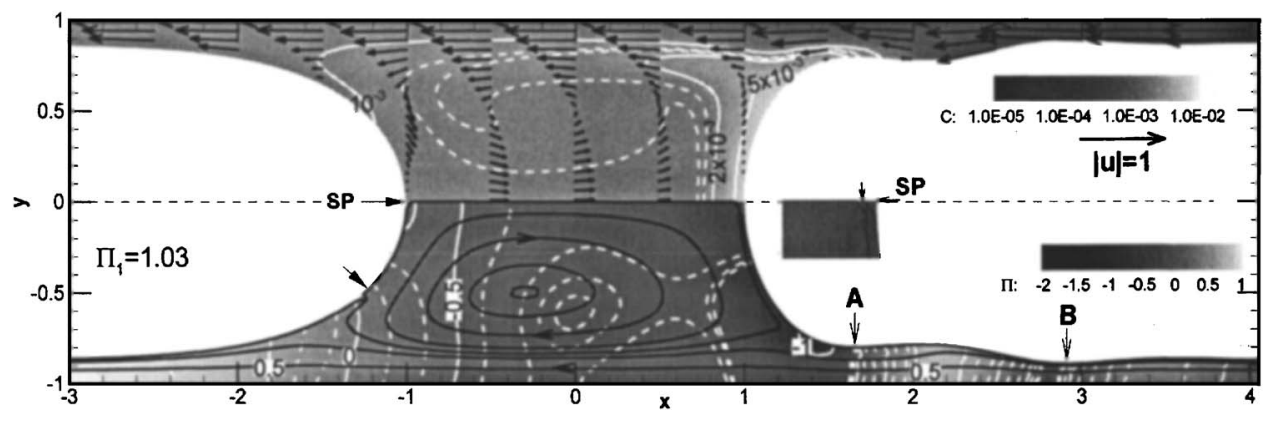

FIG. 6. Streamlines, velocity, pressure, and bulk surfactant concentration inside the liquid plug with surfactant for $L_{P}=2, \lambda=1000, \mathrm{Re}=50, \mathrm{Sc}=10$, $\mathrm{Sc}_{s}=100, K_{a}=10^{4}, K_{d}=10^{2}, \chi=10^{-2}$, $E=0.7$, and $C_{0}=10^{-4}$. 

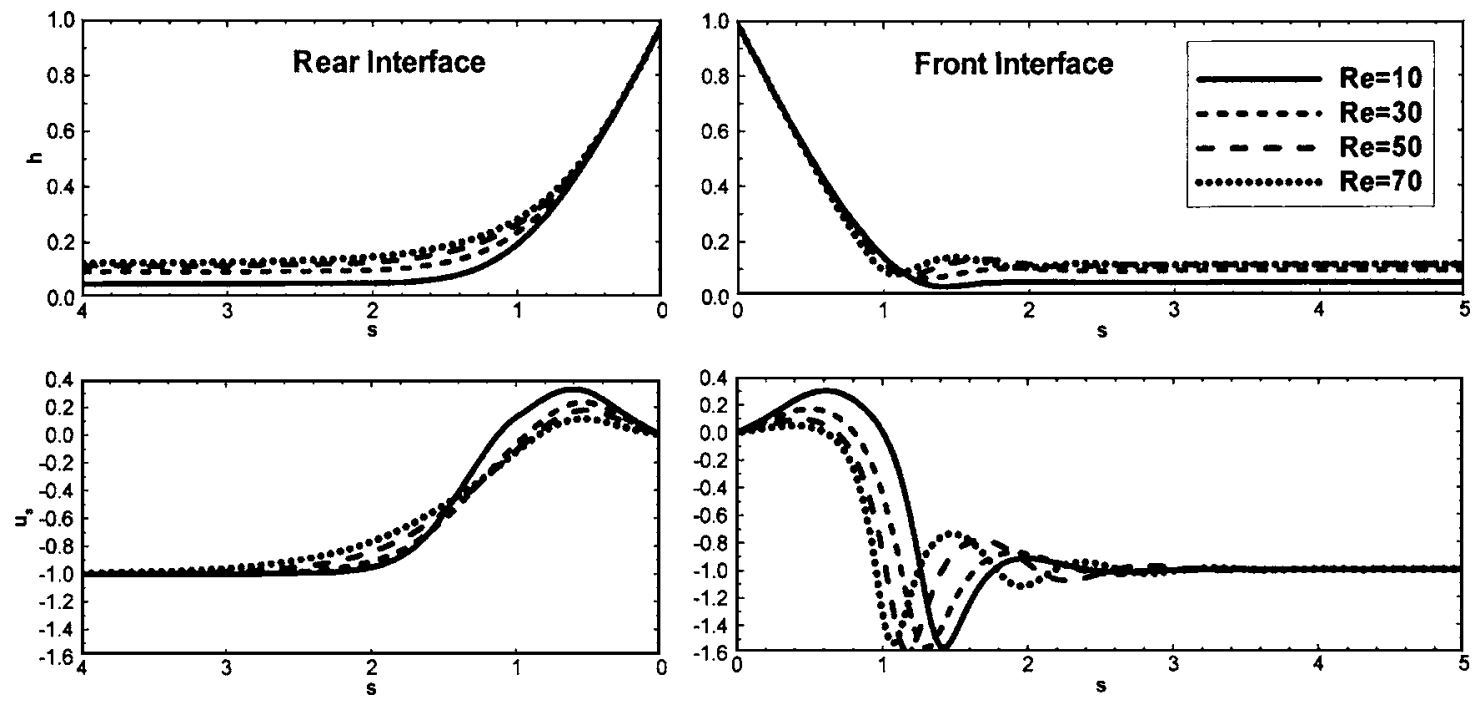

FIG. 7. The variations of the liquid thickness $h$, the interfacial surfactant concentration $\Gamma$, and the tangential component of the surface velocity $u_{s}$ along both interfaces for $\operatorname{Re}=10,30,50$, and 70 without surfactant. $L_{P}=0.5, \lambda=1000$, and $\operatorname{Re}=50$.

where this thicker film region converges on leading front precursor film (denoted by $\mathrm{B}$ ). For $\mathrm{Ca}<0.5$, a capillary wave appears in the front meniscus and the inertia effect amplifies the wave ${ }^{32,63}$ For both $L_{P}=0.5$ and $L_{P}=2$, Figs. 5 and 6, the capillary wave is overlapped with the thicker film region, which creates these local minima in thickness that are denoted by $\mathrm{A}$ and $\mathrm{B}$, and a local maximum film thickness between $A$ and $B$.

The bulk surfactant concentration is presented with constant concentration contour lines in the upper half in Figs. 5 and 6 . The concentration is maximized at the front meniscus for both $L_{P}=0.5$ and 2 because the interfacial surfactant accumulates on the front meniscus and desorbs into the bulk here. The bulk surfactant is transported towards the rear meniscus along the recirculation streamline. For Fig. 5, $L_{P}$ $=0.5$, since the velocity near the midline is fairly small, the diffusion dominates the bulk surfactant transport there, so that the concentration contour lines near the midline distribute vertically between the menisci. For Fig. $6, L_{P}=2$, since the recirculation velocity is large, the concentration contour lines in the plug core almost correspond with the streamlines, and the recirculation mixes the bulk surfactant which results in the almost uniform concentration in the core. This mixing causes a steep gradient in concentration near the front meniscus interface where the velocity is small due to the Marangoni stress. For both $L_{P}$ values, the bulk surfactant adsorbs onto the rear meniscus surface until the bulk surfactant concentration reaches equilibrium with the interfacial surfactant concentration far downstream in the trailing film.

Figures 7-9 demonstrate how the liquid thickness $h$, the interfacial surfactant concentration $\Gamma$, and the tangential component of the surface velocity $u_{s}$ vary with the plug speed, which is changed by Re. Figure 7 shows the case of $L_{P}=0.5$ without surfactant for $\operatorname{Re}=10,30,50$, and 70. Figures 8 and 9 show the cases of $L_{P}=0.5$ and 2, respectively, with surfactant for $\operatorname{Re}=10,30,50$, and 70 when $C_{0}=10^{-4}$ is prescribed at the leading front end in the precursor film. The horizontal axis $s$ is the arch length, where $s=0$ is defined at the meniscus tip on the midline. In the rear interface, negative values of $u_{s}$ are in the direction of increasing $s$ towards the rear, trailing film. In the front interface, positive values of $u_{s}$ are in the direction of increasing $s$ towards the front, precursor film. Figure 7 shows that the film thickness $h$ in the rear interface monotonically decays to $h_{1}$ as $s$ increases and the length of this transition region increases as Re increases. For the steady state, the film thickness at the right end of the precursor film has to be equal to the trailing film thickness, $h_{2}=h_{1}$, which increases with Re as shown in Fig. 3. In the front interface in Fig. 7, a capillary wave appears. As Re increases, its amplitude increases and its wavelength shortens. The film thickness minimizes where the front meniscus converges on the precursor film, which is at $s=1.18$ for Re $=50$ that is the location denoted by $\mathrm{A}$ in Fig. 4 . In the rear interface, the surface velocity in Fig. 7 shows $u_{s}=0$ at $s=0$. As $s$ increases, at first, $u_{s}$ is positive, which indicates the surface flow directs towards the midline. Thereafter, $u_{s}$ becomes negative, and then decays to $u_{s}=-1$ in the trailing film. For $\operatorname{Re}=50$, the locations of $u_{s}=0$ are at $s=0$ and 0.89 , where the stagnation points are observed at the rear meniscus midline and off the midline in Fig. 4. In the front interface, Fig. 7 shows, as $s$ increases, $u_{s}=0$ at $s=0$ and then $u_{s}$ is positive, which indicates the surface flow directs towards the precursor film. Subsequently, $u_{s}$ becomes negative and $u_{s}$ oscillates corresponding to the film thickness variation where the capillary wave is observed, and then decays to $u_{s}=-1$ in the precursor film. For $\mathrm{Re}=50$, the locations of $u_{s}=0$ are at $s=0$ and 0.69 , where the stagnation points are observed at the front meniscus midline and off the midline in Fig. 4.

When the surfactant acts on the interface, Fig. 8 for $L_{P}$ $=0.5$ shows that $h$ in the rear interface is similar to the case without surfactant as shown in Fig. 7. In the front interface, the precursor film thickness near the front meniscus is thicker than the precursor film thickness far ahead. For Re $=50, h$ for $1.2<s<1.6$ is about 1.5 times larger than $h_{2}$. This thicker film region in Fig. 8 corresponds to the film region $0.8<x<1.4$ in Fig. 5. As the plug propagates, the front me- 

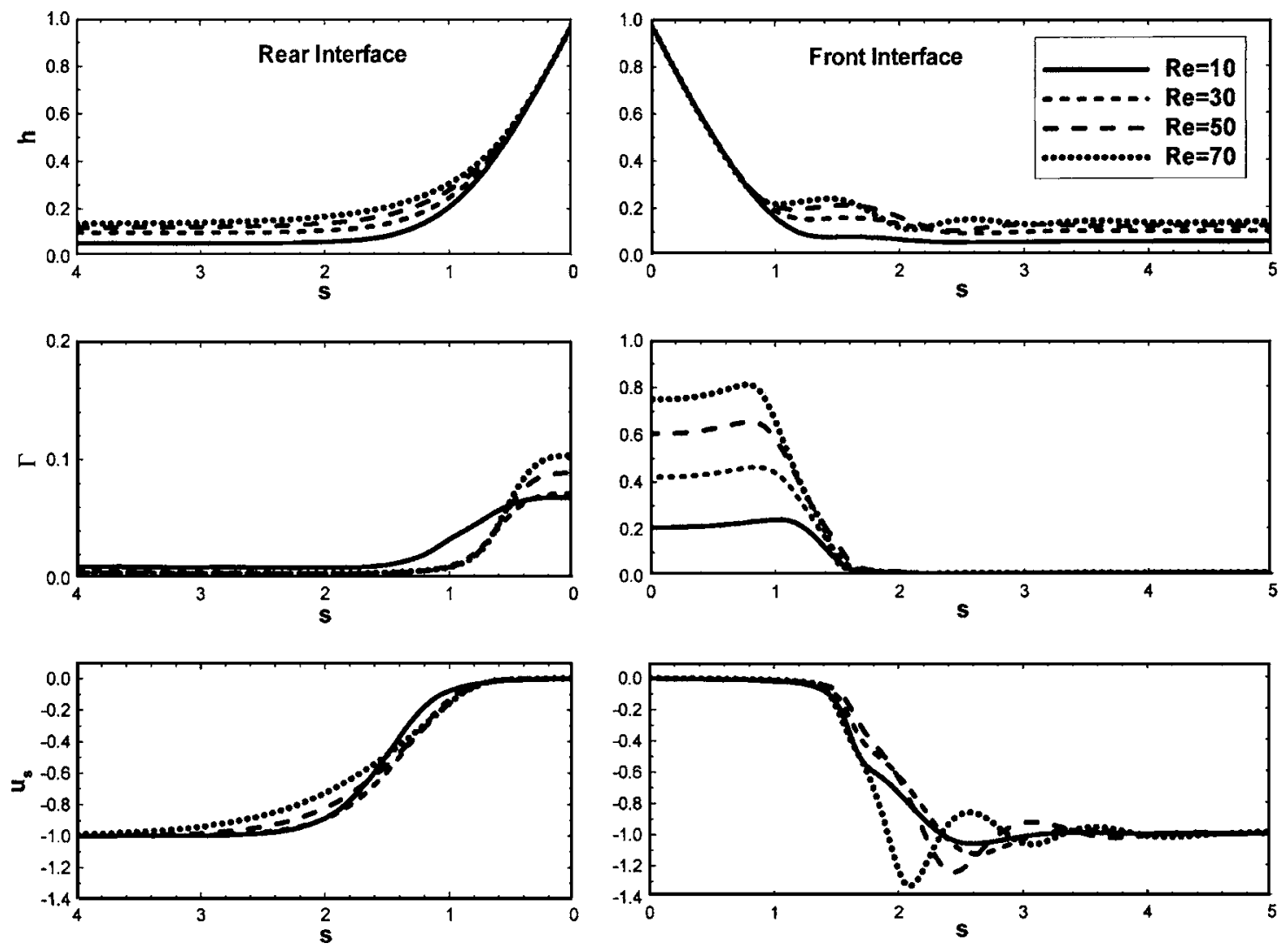

FIG. 8. The variations of the liquid thickness $h$, the interfacial surfactant concentration $\Gamma$, and the tangential component of the surface velocity $u_{s}$ along both interfaces for $\mathrm{Re}=10,30,50$, and 70. $L_{P}=0.5, \lambda=1000, \mathrm{Re}=50, \mathrm{Sc}=10, \mathrm{Sc}_{s}=100, K_{a}=10^{4}, K_{d}=10^{2}, \chi=10^{-2}, E=0.7$, and $C_{0}=10^{-4}$.

niscus sweeps up the preexisting interfacial surfactant in the precursor film. The interfacial surfactant accumulates on the front meniscus, and $\Gamma$ increases with the surface convection that increases with Re. The Marangoni stress induced by the surface tension gradient acts on the interface for the opposite direction to the flow in the precursor film, which causes the interface to be like a rigid wall. The surface velocity $u_{s}$ in this rigidified interface is fairly small. The film thickness in this region becomes thicker because of the conservation of the flow rate in the film. ${ }^{62,64}$ The surface flow in Fig. 8 shows $u_{s}=0$ only at $s=0$ on both the interfaces, which confirms the finding of one stagnation point in each interface (Fig 5). In the front interface, the curves of $u_{s}$ oscillate in the capillary wave. The peak of $u_{s}$ (negative) is observed apart from the meniscus where the thicker film region converges to the precursor film and $h$ attains the smallest value, while Fig. 7 without surfactant shows this peak where the front meniscus converges to the precursor film.

When $L_{P}$ becomes larger, Fig. 9 for $L_{P}=2$ with surfactant shows that the variations of $h, \Gamma$, and $u_{s}$ in the front meniscus have a tendency similar to that in the case of $L_{P}$ $=0.5$ shown in Fig. 8. In the rear interface, $\Gamma$ near the meniscus tip $(s=0)$ increases with $\operatorname{Re}$ more than $L_{P}=0.5$. As $\operatorname{Re}$ increases, the surfactant flux that comes in the front meniscus increases. The interfacial surfactant $\Gamma$ in the front meniscus increases with Re. Also the bulk surfactant concentration near the front meniscus increases, as Fig. 6 shows. The strong recirculation velocity transports the bulk surfactant toward the rear meniscus, which raises the bulk surfactant concentration near the rear meniscus as well as $\Gamma$ in the rear meniscus interface. The surface velocity in the rear interface in Fig. 9 indicates that for $\mathrm{Re}>10$ now two stagnation points appear on the rear meniscus in the half domain, while for $\operatorname{Re}=10$ there is just one stagnation point on the rear meniscus. When the recirculation momentum is large enough to resist the Marangoni stress that acts in the opposite direction to the recirculation flow, the recirculation is attached on the interface and there are two stagnation points on the rear meniscus in the half domain. When the amount of the surfactant increases, the Marangoni stress becomes strong and this causes the recirculation to be off the rear interface again. The details for this are shown in Sec. III B.

The pressure along the wall shown in Fig. 10 at $y=-1$ is plotted versus $x$ for $L_{P}=2$ and $C_{0}=10^{-4}$ and for $\operatorname{Re}=10,30$, 50 , and 70. At the right-end boundary, the liquid pressure is equal to the right gas pressure, $P_{2}=0$. At the left-end boundary, the liquid pressure is equal to the left gas pressure $P_{1}$, which is a part of the solution. At the left end boundary, the values of $\Pi_{\text {wall }}$ increase linearly with Re, as Fig. 2 shows that the curve of $L_{P}=2$ with the surfactant is almost a linear function of Re. The wall pressure shows a local minimum around $x=1.5$, as Fig. 6 shows that the pressure attains the smallest value where the film thickness has a local minimum. As Re increases, the capillary wave is pronounced in the precursor film near the meniscus, the wall pressure oscillates, and the wave amplitude increases. For $\operatorname{Re}=70$, the capillary wave is developed, and the region where the wall pressure attains the minimum value switches to $x=3.5$ in the precursor film. 

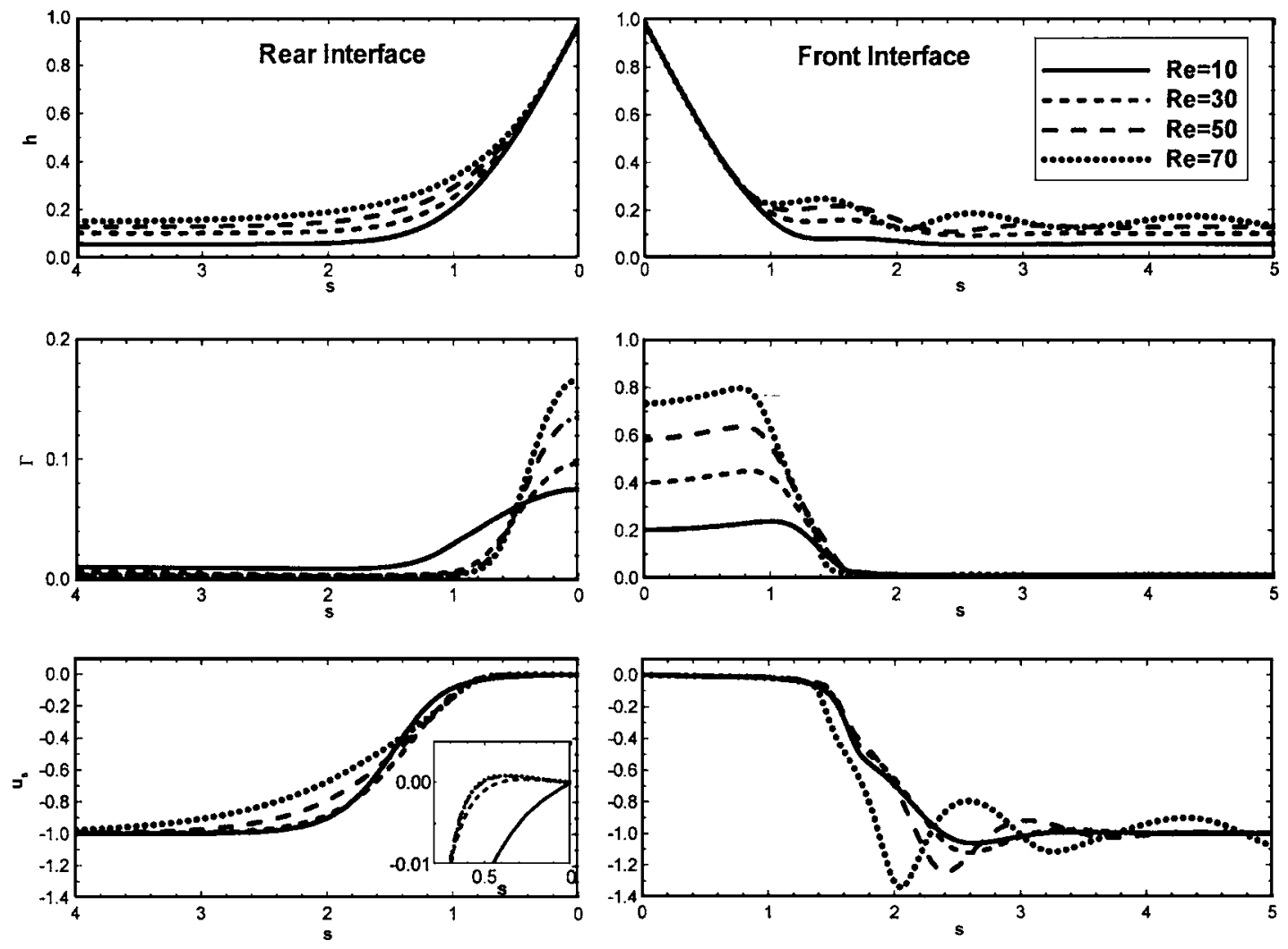

FIG. 9. The variations of the liquid thickness $h$, the interfacial surfactant concentration $\Gamma$, and the tangential component of the surface velocity $u_{s}$ along both interfaces for $\mathrm{Re}=10,30,50$, and 70. $L_{P}=2, \lambda=1000, \mathrm{Re}=50, \mathrm{Sc}=10, \mathrm{Sc}_{s}=100, K_{a}=10^{4}, K_{d}=10^{2}, \chi=10^{-2}, E=0.7$, and $C_{0}=10^{-4}$.

Figure 11 shows the dimensionless wall shear stress, where $\tau_{w}=\mathrm{Ca}(\partial u / \partial x)=\mu^{*}\left(\partial u^{*} / \partial x^{*}\right)\left(H^{*} / \sigma_{M}^{*}\right)$ at $y=-1$ is plotted versus $x$ for $L_{P}=2$ and $C_{0}=10^{-4}$, and for $\mathrm{Re}=10,30,50$, and 70. At both ends of the domain, $\tau_{w}=0$ because the flow is uniform in the film cross section. If the plug is long enough, $\tau_{w}$ in the plug core approaches the Poiseuille flow

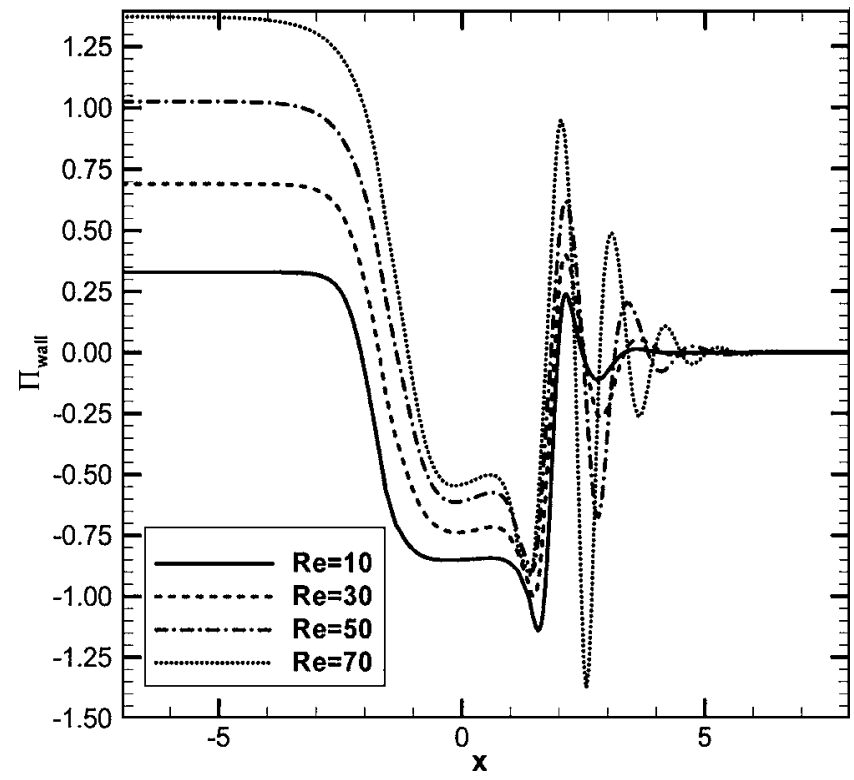

FIG. 10. The dimensionless pressure distribution along the wall $\Pi_{\text {wall }}$ vs $x$ for $\mathrm{Re}=10,30,50$, and 70. $L_{P}=2, \lambda=1000, \mathrm{Sc}=10, \mathrm{Sc}_{s}=100, K_{a}=10^{4}, K_{d}$ $=10^{2}, \chi=10^{-2}, E=0.7$, and $C_{0}=10^{-4}$. limit, $\tau_{w} \rightarrow \tau_{\infty}=3 \mathrm{Ca}\left(1-h_{1}\right)$. For $-1<x<1, \tau_{w}$ minimizes near $x=0$ and shows almost the same value as $\tau_{\infty}$. On both sides of the plug core, $\tau_{w}$ has a local peak that increases with Re. As $x$ decreases from the left peak $(x \sim 1.7), \tau_{w}$ decays to zero. The length of this transition region increases as $\operatorname{Re}$

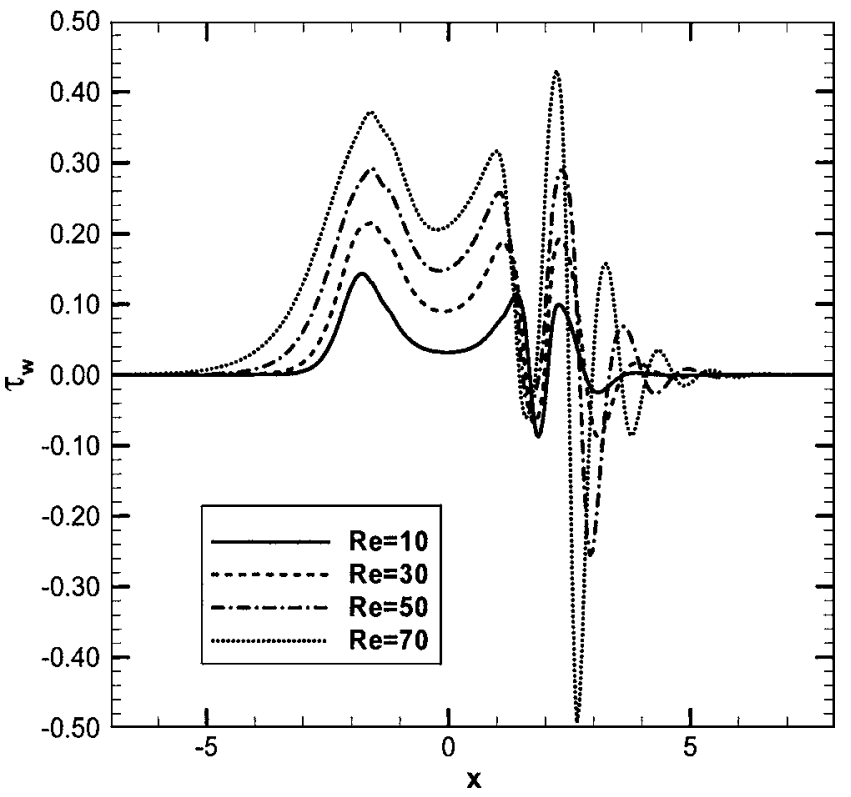

FIG. 11. The dimensionless wall shear stress distribution vs $x$ for $\operatorname{Re}=10$, 30, 50, and 70. $L_{P}=2, \lambda=1000, \mathrm{Sc}=10, \mathrm{Sc}_{s}=100, K_{a}=10^{4}, K_{d}=10^{2}, \chi$ $=10^{-2}, E=0.7$, and $C_{0}=10^{-4}$. 


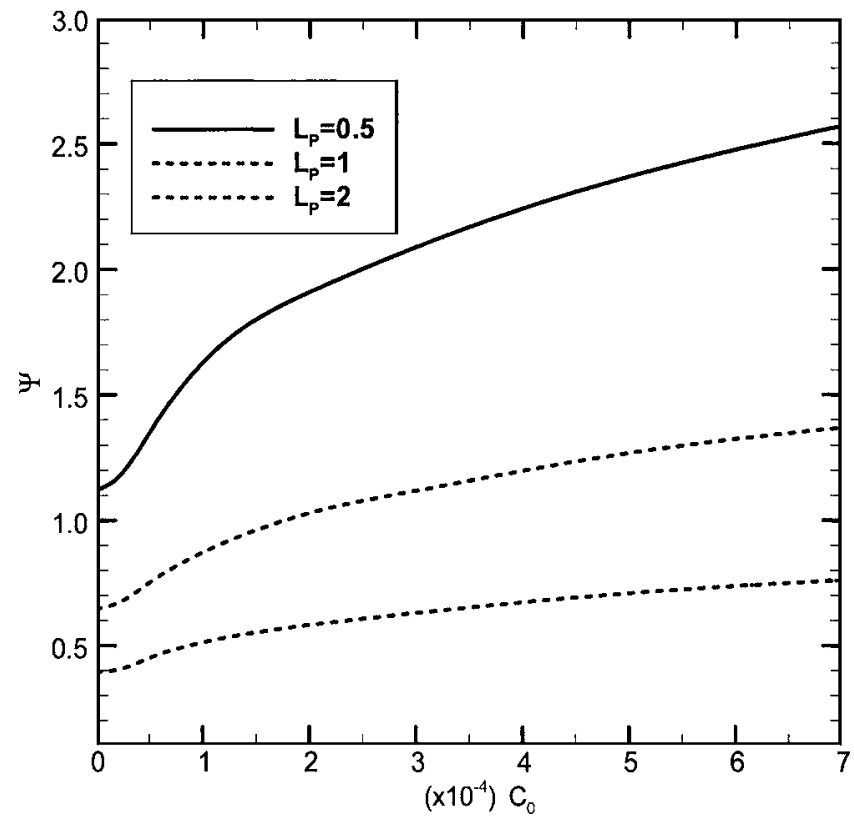

FIG. 12. The effect of the bulk surfactant concentration prescribed at the end boundary of the precursor film, $C_{0}$, on the overall pressure drop across the plug divided by the plug length, $\Psi . \lambda=1000, \mathrm{Sc}=10, \mathrm{Sc}_{s}=100, K_{a}$ $=10^{4}, K_{d}=10^{2}, \chi 10^{-2}, E=0.7$, and $\mathrm{Re}=50$.

increases. In the precursor film near the front meniscus, where the capillary wave is observed, $\tau_{w}$ oscillates with the film thickness. In the region where the front meniscus converges on the precursor film, the film thickness shows a local minimum. In this region, $\tau_{w}$ shows a local minimum, but the value is not sensitive to Re. Since the wavelength becomes shorter and its amplitude increases as Re increases, the gradient of $\tau_{w}$ in the capillary wave becomes steep for larger Re. For $\operatorname{Re}=70$, the maximum value of $\left|\tau_{w}\right|$ is observed in the precursor film ahead of the plug, $x=2.8$.

\section{B. The effect of the bulk surfactant concentration $C_{0}$}

In this section, we investigate the liquid plug propagation with changing bulk surfactant concentration prescribed at the front boundary in the precursor film, $C_{0}$. The remaining dimensionless parameters are $\mathrm{Re}=50, \lambda=1000, \mathrm{Sc}=10$, $\mathrm{Sc}_{s}=100, K_{a}=10^{4}, K_{d}=10^{2}, \chi=10^{-2}$, and $E=0.7$.

Figure 12 shows the overall pressure drop across the plug divided by the plug length, $\Psi=\left(\Delta P^{*} / L_{P}^{*}\right)\left(H^{2} / \sigma_{M}^{*}\right)$ versus $C_{0}$ for $L_{P}=0.5,1$, and 2 and at $\operatorname{Re}=50$. As $C_{0}$ increases, for a fixed $L_{P}, \Psi$ changes moderately when $C_{0}$ is very small, $\Psi$ changes rapidly for $5 \times 10^{-5}<C_{0}<10^{-4}$; thereafter, the slope of $\Psi$ becomes gentle. This indicates that the force required for the plug moving at a fixed speed increases with the surfactant concentration. Because of the steady state, the drag force acting on the channel wall balances with the force that is needed to push the plug, which is described by

$$
\left(H^{*}-h_{1}^{*}\right) \Delta P^{*}=\int_{-\infty}^{+\infty} \tau_{w}^{*} d x^{*}
$$

where $\tau_{w}^{*}=\mu^{*}\left(\partial u^{*} / \partial x^{*}\right)$ is the wall shear stress. Since $\Delta P^{*}$ increases with $C_{0}$, as Fig. 12 shows, Eq. (11) indicates that the net wall shear stress increases with $C_{0}$. As $L_{P}$ increases, $\Psi$ at a fixed $C_{0}$ decreases. As $L_{P} \rightarrow \infty$, the pressure drop near both the menisci becomes negligible compared to the viscous dissipation in the plug core. If the plug is long enough, the flow in the plug core apart from both the menisci is fully developed and, thus, the pressure drop across this region is determined by Poiseuille's law. So as $L_{P} \rightarrow \infty, \Psi$ approaches the Poiseuille flow values of $3 \operatorname{Re}\left(1-h_{1}\right) / \lambda$ and becomes independent of $C_{0}$.

Figure 13 shows the streamlines and the bulk surfactant concentration inside the plug core for $L_{P}=0.5$ and $\operatorname{Re}=50$, for four different bulk surfactant concentration prescribed far ahead of the plug, $C_{0}=0,5 \times 10^{-5}, 10^{-4}$, and $5 \times 10^{-4}$. When

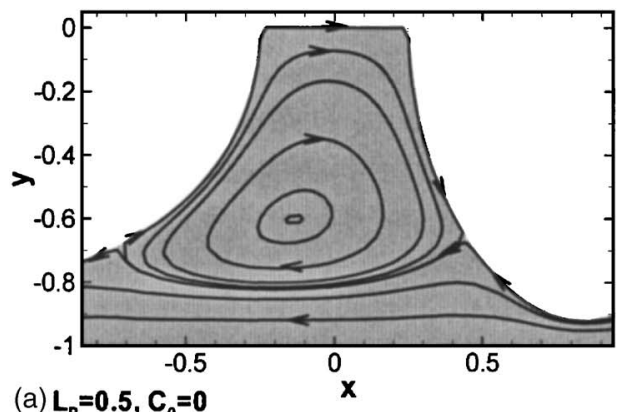

(a) $L_{P}=0.5, C_{0}=0$

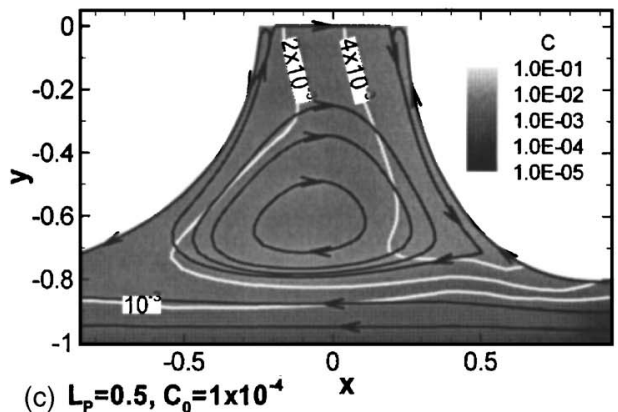

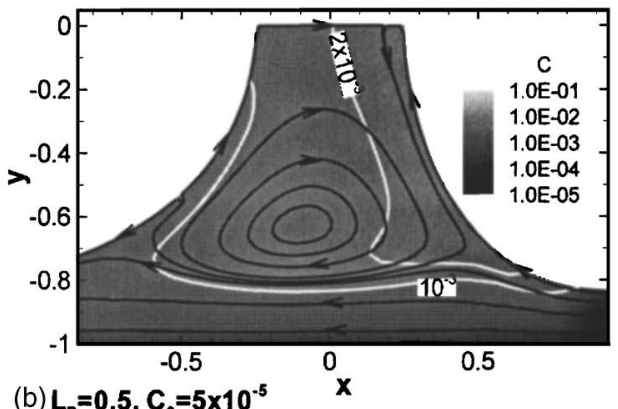

(b) $L_{P}=0.5, C_{0}=5 \times 10^{-5}$

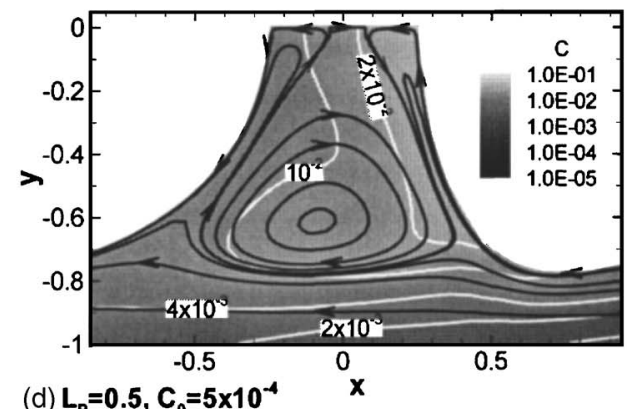

FIG. 13. The streamline patterns for four different surfactant concentrations, $C_{0}=0,5 \times 10^{-5}, 10^{-4}$, and 5 $\times 10^{-4}$, for $L_{P}=0.5, \lambda=1000, \mathrm{Re}=50$, $\mathrm{Sc}=10, \mathrm{Sc}_{s}=100, K_{a}=10^{4}, K_{d}=10^{2}$, $\chi=10^{-2}$, and $E=0.7$. 


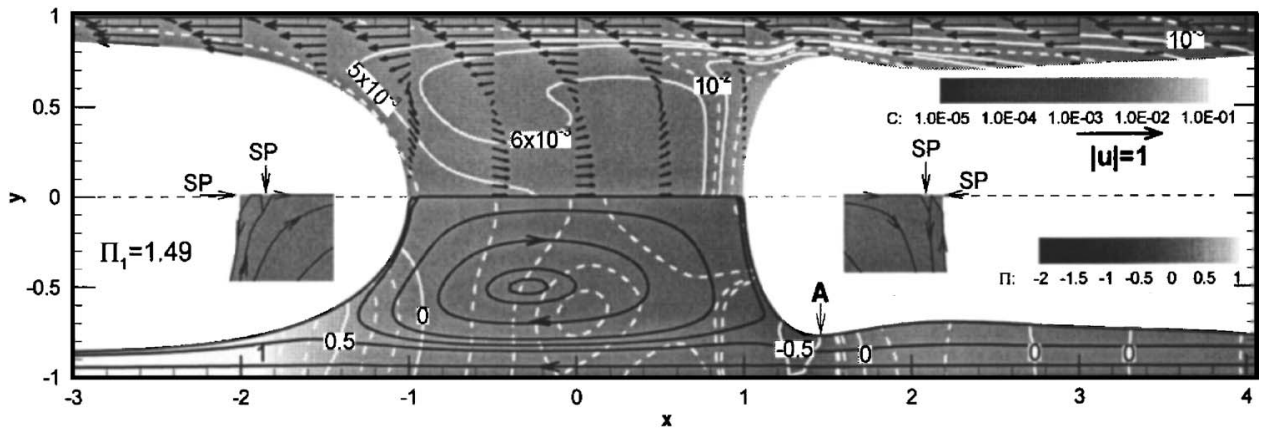

FIG. 14. Streamlines, velocity, pressure, and bulk surfactant concentration inside the liquid plug for $L_{P}=2, \lambda$ $=1000, \mathrm{Re}=50, \mathrm{Sc}=10, \mathrm{Sc}_{s}=100, K_{a}$ $=10^{4}, K_{d}=10^{2}, \chi=10^{-2}, E=0.7$, and $C_{0}=5 \times 10^{-4}$. The upper half contour plot represents the bulk surfactant concentration, and the lower half contour plot represents the pressure field. no surfactant acts on the interface, Fig. 13(a), $C_{0}=0$ shows that the recirculation zone attaches to both the front and the rear interfaces and there are four stagnation points on the interfaces in the half domain. When $C_{0}$ increases to $C_{0}=5$ $\times 10^{-5}$, Fig. 13(b) shows that the bulk surfactant near the front meniscus increases and the maximum $C$ is $4.1 \times 10^{-3}$. The streamline on the front interface climbs up to the front meniscus tip; thus, there is only one interfacial stagnation point at the tip in the front interface and a stagnation point appears inside the plug on the midline, while the two stagnation points remain on the rear interface. As $C_{0}$ increases, the streamlines rear the rear meniscus change as those near the front meniscus. For Fig. 13(c), $C_{0}=10^{-4}$, the recirculation zone is off both the front and the rear interfaces and four stagnation points appear on the midline. The bulk surfactant concentration for Fig. 13(c) increases approximately twice as much as for Fig. 13(b) and the maximum $C$ is $8.4 \times 10^{-3}$ on the front interface. For Fig. $13(\mathrm{~d}), C_{0}=5 \times 10^{-4}$, the maxi- mum $C$ is now $3.8 \times 10^{-2}$ near the front interface. The streamline shows a similar pattern as in Fig. 13(c), but the two stagnation points that are off the interface on the midline are now close together.

When $C_{0}=10^{-4}$, for Fig. $6, L_{P}=2$, two stagnation points remain on the rear interface while for Fig. $5, L_{P}=0.5$, all the stagnation points appear on the midline. When $C_{0}=5 \times 10^{-4}$, Fig. 14 shows that the streamline for $L_{P}=2$ now changes to a similar pattern as in Fig. 5; the streamline on both the interfaces climbs up to the meniscus tip and all stagnation points appear on the midline. The bulk surfactant concentration attains the maximum value of $C=2.6 \times 10^{-2}$ on the front interface. The precursor film near the front meniscus is thick for a longer length than in Fig. 6. The surface velocity in this region is almost zero and the wall shear rate is large. The details for this are shown later.

Figure 15 shows $h, \Gamma$, and $u_{s}$ along both the interfaces for $L_{P}=2$ and $\mathrm{Re}=50$, and for four different surfactant con-
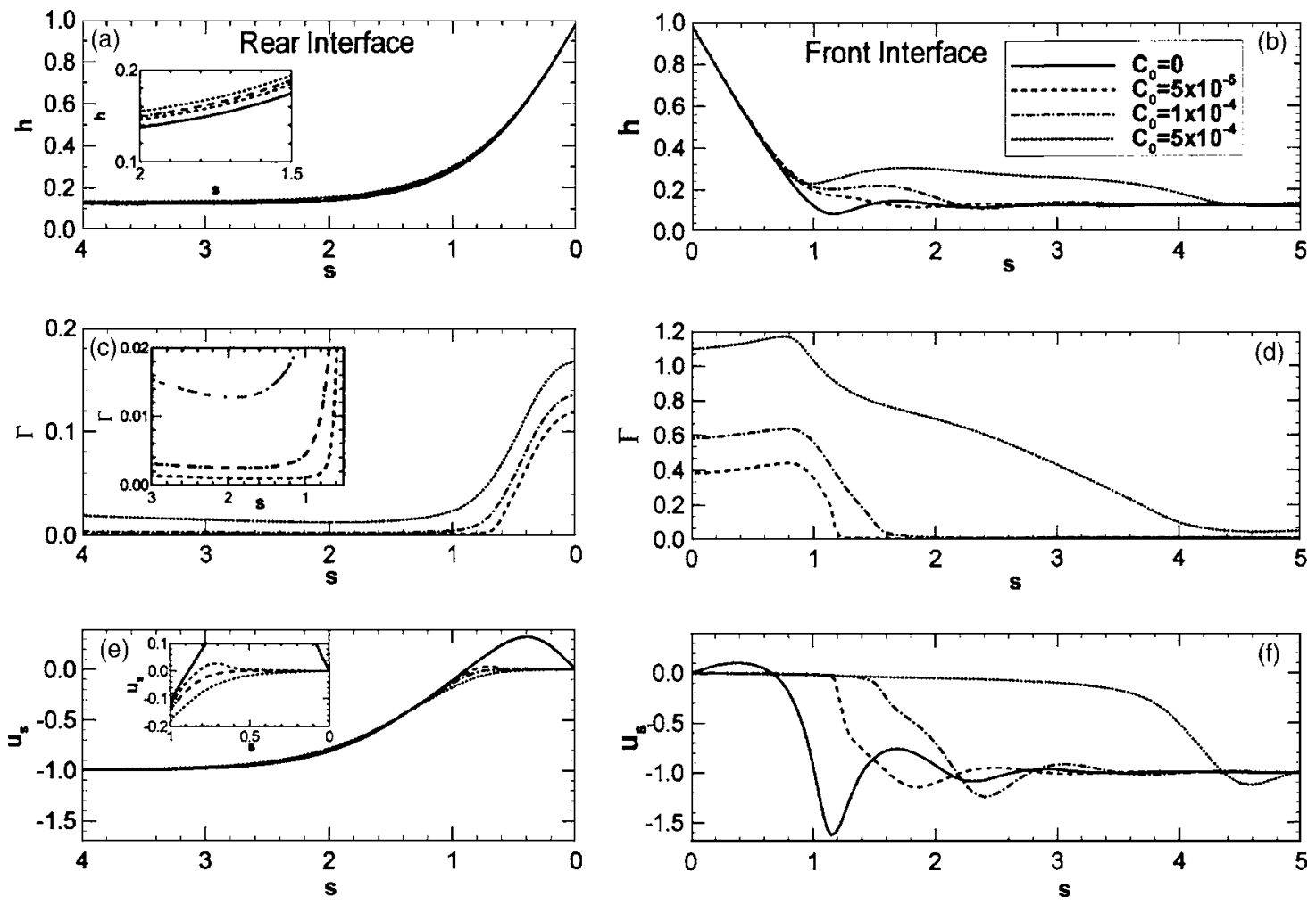

FIG. 15. The effect of the surfactant concentration on the liquid thickness $h$, the interfacial surfactant concentration $\Gamma$, and the tangential component of the surface velocity $u_{s}$ along the both interfaces. $L_{P}=2, \lambda=1000, \mathrm{Re}=50, \mathrm{Sc}=10, \mathrm{Sc}_{s}=100, K_{a}=10^{4}, K_{d}=10^{2}, \chi=10^{-2}$, and $E=0.7$. 
centrations, $C_{0}=0,5 \times 10^{-5}, 1 \times 10^{-4}$, and $5 \times 10^{-4}$. The film thickness $h$ in the rear interface [Fig. 15(a)] monotonically decreases with $s$ to the trailing film thickness $h_{1}$ that increases with $C_{0}$. The film thickness $h$ in the front interface [Fig. 15(b)] for $C_{0}=0$ is smallest at $s=1.1$, but when a small amount of surfactant exists in the precursor film, for $C_{0}=5$ $\times 10^{-5}$, this minimum in $h$ disappears. Due to the accumulation of the surfactant on the front meniscus surface, $\Gamma$ arises in the front meniscus that causes a steep gradient in $\sigma$ for $1<s<1.2$ as shown in Fig. 15(d). In this region, the film is thicker because the Marangoni stress causes $u_{s}$ in this region to be small. As $C_{0}$ increases, the Marangoni stress affects larger region of the precursor film interface; for $C_{0}=1$ $\times 10^{-4}, h$ for $1.2<s<1.6$ is about 1.7 times thicker than $h_{2}$. For $C_{0}=5 \times 10^{-4}$, this region is longer and $h$ for $1.7<s$ $<3.5$ is about twice thicker than $h_{2}$. The rear interfacial surfactant concentration $\Gamma$ [Fig. 15(c)], increases with $C_{0}$ and shows a maximum value at the meniscus tip, $s=0 . \Gamma$ decreases with $s$ and minimizes around $s=2$ in the trailing film, then increases to the equilibrium values that are $\Gamma_{\text {eq }}=4.98$ $\times 10^{-3}, 9.90 \times 10^{-3}$, and $4.76 \times 10^{-2}$ for the cases $C_{0}=5$ $\times 10^{-5}, 1 \times 10^{-4}$, and $5 \times 10^{-4}$, respectively. The change in $\Gamma$ reflects the change in the surface tension; the surface tension $\sigma$ minimizes at $s=0$ and increases with $s$. The gradient in $\sigma$ induces the Marangoni stress that affects the surface flow $u_{s}$. In the front meniscus [Fig. 15(d)] the surfactant accumulates for $0 \leqslant s<0.8$ and $\Gamma$ here increases with $C_{0}$. Attains $\Gamma$ a maximum around $s=0.8$. The Marangoni stress acts towards the meniscus tip for $0 \leqslant s<0.8$, where $\Gamma$ decreases from $s$ $=0.8$ towards the tip. For $s>0.8$, as $s$ increases from $s=0.8$, $\Gamma$ decreases with a steep gradient until $\Gamma$ reaches an equilibrium value with $C_{0}$. The Marangoni stress acts towards the precursor film here. In this region, where the front meniscus converges to the precursor film, the Marangoni stress causes the surface velocity $u_{s}$ to be small. The surface velocity $u_{s}$ in the rear meniscus [Fig. 15(e)] for $C_{0}=0$ is negative for 0 $<s<0.9$; there are two stagnation points at $s=0$ and 0.9 . For $C_{0} \geqslant 5 \times 10^{-5}$, the Marangoni stress makes the interface less mobile and $u_{s}$ in the rear meniscus small, but there are still two stagnation points on the interface for $C_{0} \leqslant 10^{-4}$. When $C_{0}=5 \times 10^{-4}$, there is one stagnation point on the rear interface. In the front meniscus [Fig. 15(f)], $u_{s}$ for $C_{0}=0$ is positive for $0<s<0.7$; there are two stagnation points at $s=0$ and 0.7. For $C_{0} \geqslant 5 \times 10^{-5}, u_{s}$ is negative in whole range of $s$ and $u_{s}$ is almost zero from the meniscus tip to the region where $h$ is thicker than $h_{2}$.

For $C_{0}=5 \times 10^{-4}, \Gamma>1$ for $0 \leqslant s \leqslant 1$; the interfacial surfactant concentration exceeds the maximum equilibrium concentration. In this regime, the surfactant layer is compressed due to the surface convection. When the interfacial surfactant concentration attains the maximum dynamic concentration, the surfactant layer will buckle or collapse and will form micelles. ${ }^{38,39,55-61}$ This concentration is approximately $\Gamma_{\max }^{*} / \Gamma_{\infty}^{*}=1.1-1.2 .^{38,39}$ Figure $15(\mathrm{~d})$ shows $\Gamma$ for $C_{0}=5$ $\times 10^{-4}$ attains the maximum value of $\Gamma=1.17$ at $s=0.76$. When $C_{0}=7 \times 10^{-4}$ is prescribed, the maximum interfacial surfactant concentration becomes $\Gamma>1.4$ in the front meniscus. The interfacial surfactant might form multilayer in this

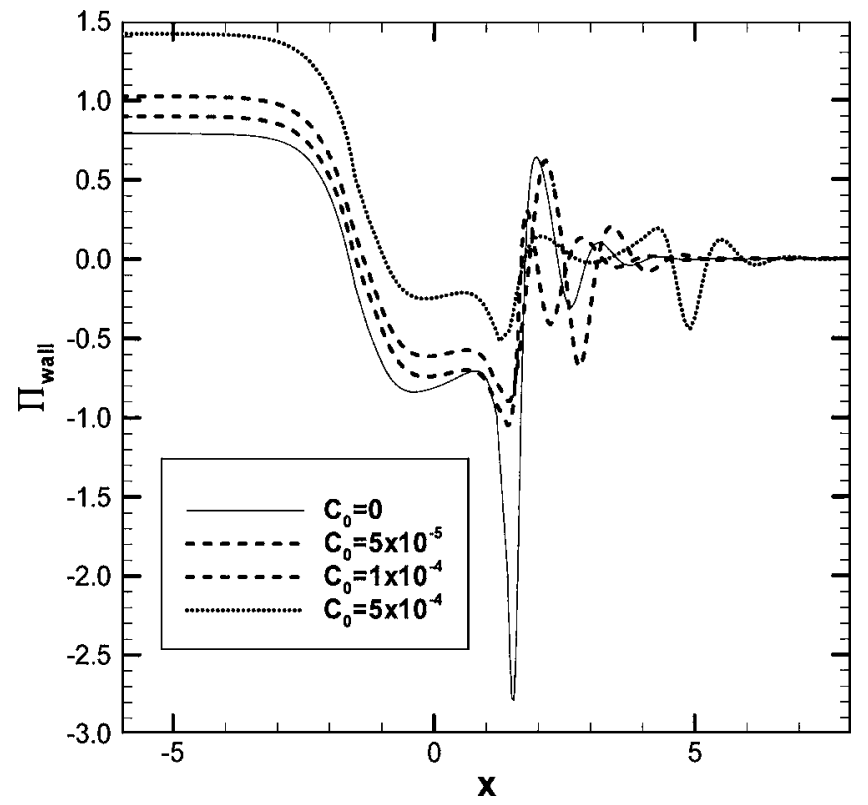

FIG. 16. The effect of the surfactant concentration on the wall pressure. $L_{P}=2, \lambda=1000, \mathrm{Re}=50, \mathrm{Sc}=10, \mathrm{Sc}_{s}=100, K_{a}=10^{4}, K_{d}=10^{2}, \chi=10^{-2}$, and $E=0.7$.

region. The second layer will form micelles and may detach from the interface by the bulk convection, which is not considered in the present study.

The pressure along the wall, $\Pi_{\text {wall }}$, is shown in Fig. 16, where the dimensionless pressure, $\Pi=\mathrm{Ca} p=p^{*} /\left(\sigma_{M}^{*} / H^{*}\right)$ at $y=-1$, is plotted versus $x$ for $L_{P}=2$ and $\operatorname{Re}=50$, and for $C_{0}$ $=0,5 \times 10^{-5}, 1 \times 10^{-4}$, and $5 \times 10^{-4}$, which are the bulk surfactant concentrations prescribed at the boundary of the precursor film. The values of $\Pi_{\text {wall }}$ at the left end boundary increase with $C_{0}$, as Fig. 12 shows that the curve of $L_{P}=2$ is monotonically increasing with $C_{0}$. The wall pressure is negative in the plug core and shows a local minimum around $x$ $=1.5$, as Figs. 6 and 14 show that the pressure attains the smallest value where the film thickness has a local minimum. As $C_{0}$ increases, the magnitude of this local peak in $\Pi_{\text {wall }}$ decreases, because the film thickness in this region becomes thicker.

The effect of the surfactant concentration on the wall shear stress is shown in Fig. 17, where the dimensionless wall shear stress, $\tau_{w}=\mu^{*}\left(\partial u^{*} / \partial x^{*}\right)\left(H^{*} / \sigma_{M}^{*}\right)$ is plotted versus $x$ for $L_{P}=2$ and $\mathrm{Re}=50$, and for $C_{0}=0,5 \times 10^{-5}, 1 \times 10^{-4}$, and $5 \times 10^{-4}$. In the rear half of the plug, $x<0, \tau_{w}$ for the four different $C_{0}$ values shows similar behavior, because the shape of the rear interface is not sensitive to $C_{0}$ for the range $0 \leqslant C_{0} \leqslant 5 \times 10^{-4}$, and also the surface velocity in the rear film region, $s>1$, does not change much, as shown in Figs. $15(\mathrm{a})$ and $15(\mathrm{e})$. In the front part, however, $\tau_{w}$ changes significantly with the value of $C_{0}$. For the case without surfactant, $C_{0}=0, \tau_{w}$ shows a sharp negative peak around $x=1.7$ where the film thickness is smallest in the capillary wave. As $C_{0}$ increases, this sharp peak weakens. For $C_{0} \geqslant 1 \times 10^{-4}$, the maximum of $\left|\tau_{w}\right|$ is observed in the rear transition region. For the case $C_{0}=5 \times 10^{-4}, \tau_{w}$ keeps a positive value in the precursor film near the front meniscus where the film is thicker. 


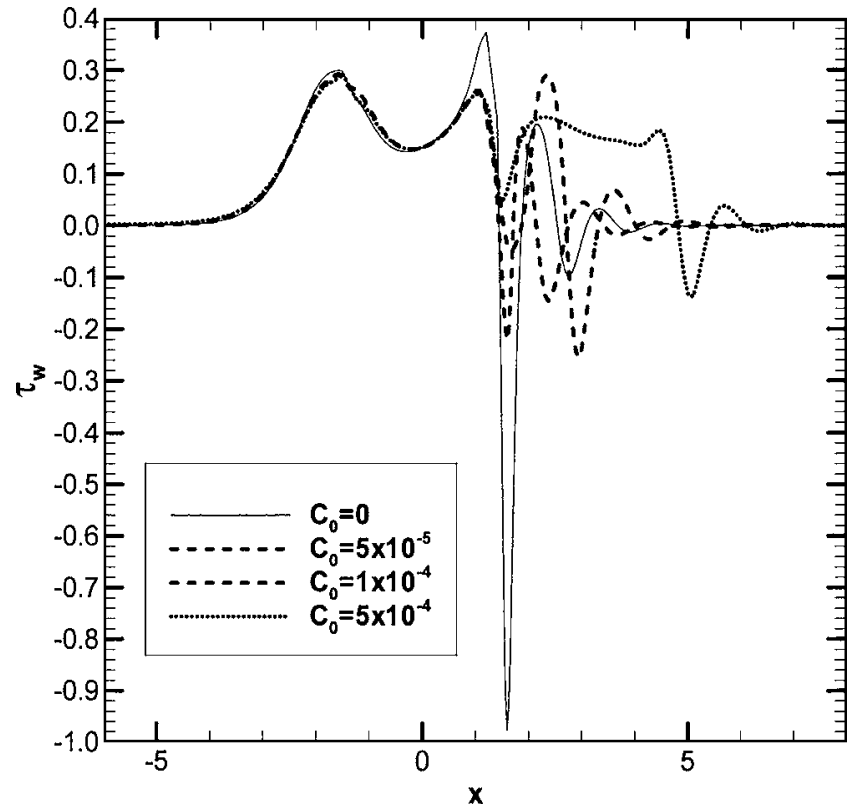

FIG. 17. The effect of the surfactant concentration on the wall shear stress. $L_{P}=2, \lambda=1000, \mathrm{Re}=50, \mathrm{Sc}=10, \mathrm{Sc}_{s}=100, K_{a}=10^{4}, K_{d}=10^{2}, \chi=10^{-2}$, and $E=0.7$.

Figure 18 shows the velocity and the pressure profiles in the precursor film near the front meniscus for $L_{P}=2$ and $\mathrm{Re}=50$, and for $C_{0}=0,5 \times 10^{-5}, 1 \times 10^{-4}$, and $5 \times 10^{-4}$. For $C_{0}=0$, the velocity is uniform in $y$ while the film thickness is uniform in $x$. In the cross section where the film is thinnest at $x=1.58$, the fluid velocity at the film surface is faster than the velocity at the wall to conserve the flow rate in the film, $-h_{2}$. The wall shear rate attains a large negative value; thus, $\tau_{w}$ has a negative peak in this region, as shown in Fig. 17 for $C_{0}=0$. When a small amount of surfactant preexists on the leading film, for $C_{0}=5 \times 10^{-5}$, the Marangoni stress results in nearly zero surface velocity for $x<1.6$, which causes the film to thicken in order to conserve the flow rate $-h_{2}$ (Ref. 62) and reduces the peak of $\tau_{w}$. For $C_{0}=1 \times 10^{-4}$ and 5 $\times 10^{-4}$, in the thick film region, the velocity has a gradient in $y$ because the surface velocity is almost zero, which causes a positive value of $\tau_{w}$, as shown in Fig. 17.

\section{DISCUSSION}

A thin liquid layer coating inside an airway is unstable and tends to form a meniscus because of a capillary instability. ${ }^{2-4}$ When the amount of surfactant acting on airliquid interface is reduced, this tendency can be increased ${ }^{5}$ and, if enough liquid exists, it forms a plug. Liquid plugs are also instilled into airways in some medical treatments such as surfactant replacement therapy, partial liquid ventilation, and drug delivery. Mechanical stresses induced by propagation of the liquid plug may cause pulmonary epithelial cell damage. ${ }^{6}$ Particularly, in the front meniscus, when there is no surfactant present, the wall pressure and the wall shear stress have sharp peaks where the liquid film is thinnest. ${ }^{32}$ These
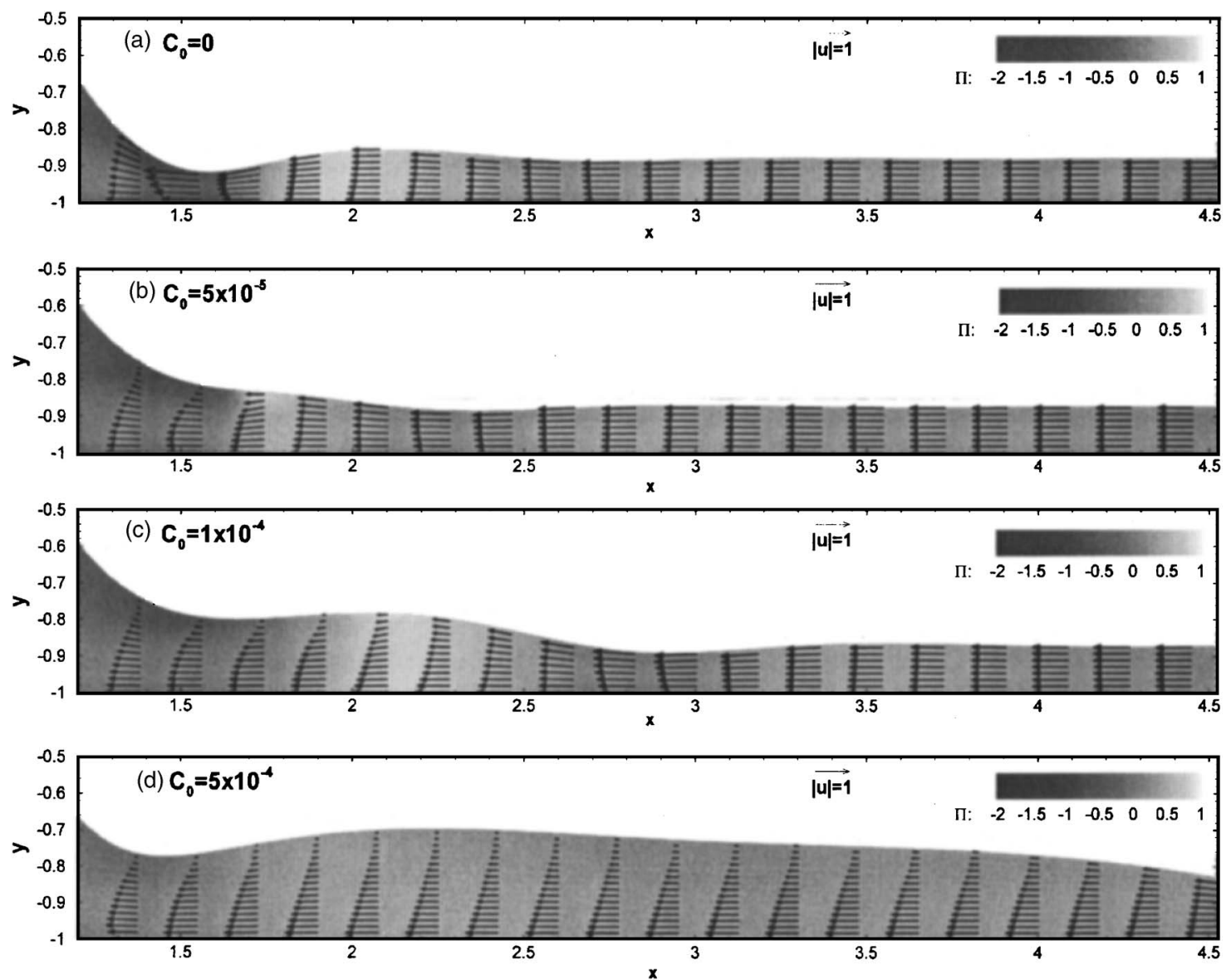

FIG. 18. The velocity and pressure profiles in the precursor film near the front meniscus for $C_{0}=0,5 \times 10^{-5}, 10^{-4}$, and $5 \times 10^{-4} . L_{P}=2, \lambda=1000, \operatorname{Re}=50$, $\mathrm{Sc}=10, \mathrm{Sc}_{s}=100, K_{a}=10^{4}, K_{d}=10^{2}, \chi=10^{-2}$, and $E=0.7$. 
peaks increase with the plug propagation speed and a developing capillary wave causes these stresses to oscillate corresponding to the oscillation of the film thickness. In this study, we have shown that if a surfactant acts on the interface, the surfactant reduces the peaks of wall pressure and wall shear stress in the front meniscus. As the plug propagates, the front meniscus sweeps the preexisting interfacial surfactant on the precursor film. When the desorption rate is slower than the interfacial surfactant's convective flux rate, the surfactant accumulates on the front meniscus interface, which results in a gradient in surface tension on the precursor film near the front meniscus. The Marangoni stress induced by the surface tension gradient causes the liquid surface to be less mobile. Since the interface becomes like a ridged wall, the film thickness must be thicker than the leading film thickness to conserve the flow rate. ${ }^{62}$ Since the peaks of the mechanical stresses occur due to narrowing of the film thickness, the increase of the minimum film thickness weakens these stresses.

As the surfactant concentration prescribed at the precursor film far ahead of the plug increases, the region where the interface is less mobile increases. This causes the force required for plug propagation to increase. The overall pressure drop through the plug increases with the surfactant concentration, as shown in Fig. 12. Since under the steady-state condition, the drag force balances with the total force to push the plug, the total tangential stress acting on the wall increases with the surfactant concentration. The surfactant reduces the peak of the wall shear stress, but the total wall shear stress increases with the surfactant concentration. When a liquid plug is driven by a constant pressure difference between the front and the rear air phases, the presence of surfactant will reduce the plug propagation speed. Since the trailing film thickness increases with the plug propagation speed, we can infer that decreasing of the trailing film thickness delays or avoids plug rupture when the plug propagates with losing mass.

A pair of recirculation regions appears in the plug core for all cases in this study. The magnitude of recirculation velocity decreases as the plug length decreases. For a finite $\mathrm{Re}$, if the plug is long enough, centrifugal forces due to the strong recirculation velocity cause the pressure near the center of the recirculation to be small. The Marangoni stress acts to reduce the surface velocity and changes the locations of the stagnation points. When no surfactant exists, there are four stagnation points in the half domain: two on each front and rear menisci, one at the midline, the other off of the midline on each interface. As $C_{0}$ increases, the streamline from the precursor film begins to climb up to the front meniscus tip. The stagnation points appear at the front meniscus tip and near the tip on the midline inside the plug. Subsequently, as $C_{0}$ increases, the streamline pattern in the rear meniscus also changes in a manner similar to that of the front meniscus. The stagnation points in the rear meniscus appear at the rear meniscus tip and near the tip on the midline inside the plug. This change in the streamline pattern occurs at smaller $C_{0}$ when the plug length is shorter.

In Sec. III B, $C_{0}=0,5 \times 10^{-5}, 1 \times 10^{-4}$, and $5 \times 10^{-4}$ are employed for the bulk surfactant concentration prescribed at the end boundary in the precursor film. The equilibrium interfacial surfactant concentrations with these values are $\Gamma_{\text {eq }}$ $=0,4.98 \times 10^{-3}, 9.90 \times 10^{-3}$, and $4.76 \times 10^{-2}$; the corresponding surface tensions are $\sigma=1,0.999,0.993$, and 0.967, respectively. In this study, we have shown that even if the amount of preexisting surfactant is small, the surfactant reduces the peaks of mechanical stresses. In this study, the computations are performed in the regime $K_{a}>K_{d}$; the adsorption rate is much faster than the desorption rate, which is one of the characteristics of the pulmonary surfactant. This condition requires the net desorption rate to be slow and enhances the accumulation of the surfactant at the site where the net surface convective flux is large. In the present numerical model, the maximum bulk surfactant concentration must be less than the critical micelle concentration and the interfacial surfactant concentration must be less than the maximum dynamic concentration. Due to these limits, we cannot use a large value of $C_{0}$ in the present study. For the model of surfactant replacement therapy, the bulk fluid will be a suspension colloid of micelles, and the fluid viscosity has a non-Newtonian behavior, of which the yield stress depends on the concentration of micelles. ${ }^{65}$ To investigate the plug propagation with a much higher surfactant concentration, it is necessary to add micelle production and its transport process to the computational model.

\section{ACKNOWLEDGMENTS}

This work was supported by NIH grants under Grant Nos. HL41126 and HL64373, and NASA grants under Grant Nos. NAG3-2196 and NAG3-2740.

${ }^{1}$ M. E. Avery and J. Mead, "Surface properties in relation to atelectasis and hyaline membrane disease," Am. J. Dis. Child 97, 517 (1959).

${ }^{2}$ J. M. B. Hughes, D. Y. Rosenzweig, and P. B. Kivitz, "Site of airway closure in excised dog lungs: Histologic demonstration," J. Appl. Physiol. 29, 340 (1970).

${ }^{3}$ P. T. Macklem, D. F. Proctor, and J. C. Hogg, "The stability of peripheral airways," Respir. Physiol. 8, 191 (1970).

${ }^{4}$ R. D. Kamm and R. C. Schroter, "Is airway closure caused by a thin liquid instability?" Respir. Physiol. 75, 141 (1989).

${ }^{5}$ D. Halpern and J. B. Grotberg, "Fluid-elastic instabilities of liquid-lined flexible tubes," J. Fluid Mech. 244, 615 (1992).

${ }^{6}$ A. M. Bilek, K. C. Dee, and D. P. Gaver, "Mechanisms of surface-tensioninduced epithelial cell damage in a model of pulmonary airway reopening," J. Appl. Physiol. 94, 770 (2003).

${ }^{7}$ W. Long, T. Thompson, H. Sundell, and R. Schumacher, F. Volberg, and R. Guthrie, "Effects of two rescue doses of a synthetic surfactant on mortality rate and survival without broncho-pulmonary dysplasia in 700- to 1350-gram infants with respiratory distress syndrome. The American Exosurf Neonatal Study Group I,” J. Pediatr. (St. Louis) 118, 595 (1991).

${ }^{8}$ B. Robertson, "Surfactant replacement therapy for severe neonatal respiratory distress syndrome: An international randomized clinical trial," J. Pediatr. (St. Louis) 82, 683 (1988).

${ }^{9}$ H. Yapicioglu, D. Yildizdas, I. Bayram, Y. Sertdemir, and H. L. Yilmaz, "The use of surfactant in children with acute respiratory distress syndrome: Efficacy in terms of oxygenation, ventilation and mortality," Pulm. Pharmacol. Ther. 16, 327 (2003).

${ }^{10}$ M. D. Salvia-Roiges, X. Carbonell-Estrany, J. Figueras-Aloy, and J. M. Rodriguez-Miguelez, "Efficacy of three treatment schedules in severe meconium aspiration syndrome," Acta Pediatr. Esp. 93, 60 (2004).

${ }^{11}$ E. M. Zola, J. H. Gunkel, R. K. Chan, M. O. Lim, I. Knox, B. H. Feldman, S. E. Denson, B. S. Stonestreet, B. R. Mitchell, M. M. Wyza, K. J. Bennett, and A. J.Gold, "Comparison of 3 dosing procedures for administration of bovine surfactant to neonates with respiratory-distress syndrome," J. Pediatr. (St. Louis) 122, 453 (1993). 
${ }^{12}$ R. B. Hirschl, R. Tooley, A. C. Parent, K. Johnson, and R. H. Bartlett, "Improvement of gas exchange, pulmonary function, and lung injury with partial liquid ventilation. A study model in a setting of severe respiratory failure," Chest 108, 500 (1995).

${ }^{13}$ T. H. Shaffer and M. R. Wolfson, "Liquid ventilation: An alternative ventilation strategy for management of neonatal respiratory distress," Eur. J. Pediatr. 155, S30 (1996).

${ }^{14}$ H. P. Baden, J. D. Mellema, S. L. Bratton, P. P. O'Rourke, and J. C. Jackson, "High-frequency oscillatory ventilation with partial liquid ventilation in a model of acute respiratory failure," Crit. Care Med. 25, 299 (1997).

${ }^{15}$ C. L. Leach, J. S. Greenspan, S. D. Rubenstein, T. H. Shaffer, M. R. Wolfson, J. C. Jackson, R. DeLemos, and B. P. Fuhrman, "Partial liquid ventilation with perflubron in premature infants with severe respiratory distress syndrome. The LiquiVent Study Group," N. Engl. J. Med. 335, 761 (1996).

${ }^{16}$ K. Mikawa, K. Nishina, Y. Takao, and H. Obara, "Efficacy of partial liquid ventilation in improving acute lung injury induced by intratracheal acidified infant formula: Determination of optimal dose and positive endexpiratory pressure level," Crit. Care Med. 32, 209 (2004).

${ }^{17}$ C. W. Choi, J. H. Hwang, Y. S. Chang, and W. S. Park, "Combined effect of low-dose nitric oxide gas inhalation with partial liquid ventilation on hemodynamics, pulmonary function, and gas exchange in acute lung injury of newborn piglets," J. Korean Med. Sci. 18, 813 (2003).

${ }^{18}$ P. N. Cox, H. Frndova, O. Karlsson, S. Holowka, and C. A. Bryan, "Fluorocarbons facilitate lung recruitment," Intensive Care Med. 29, 2297 (2003).

${ }^{19}$ D. J. Weiss, L. Bonneau, and D. Liggitt, "Use of perfluorochemical liquid allows earlier detection of gene expression and use of less vector in normal lung and enhances gene expression in acutely injured lung," Mol. Ther. 3, 734, Part 1 (2001).

${ }^{20}$ K. Nakazawa, K. Yokoyama, Y. Matsuzawa, K. Makita, and K. Amaha, "Pulmonary administration of prostacyclin (PGI(2)) during partial liquid ventilation in an oleic acid-induced lung injury: inhalation of aerosol or intratracheal instillation?" Intensive Care Med. 27, 243 (2001).

${ }^{21}$ E. W. Dickson, S. O. Heard, T. E. Tarara, J. G. Weers, A. B. Brueggemann, and G. V. Doern, "Liquid ventilation with perflubron in the treatment of rats with pneumococcal pneumonia," Crit. Care Med. 30, 393 (2002).

${ }^{22}$ J. Yu and Y. W. Chien, "Pulmonary drug delivery: Physiologic and mechanistic aspects," Crit. Rev. Ther. Drug Carrier Syst. 14, 395 (1997).

${ }^{23}$ E. Raczka, J. E. Kukowska-Latallo, M. Rymaszewski, C. Chen, and J. R. Baker, Jr., "The effect of synthetic surfactant Exosurf on gene transfer in mouse lung in vivo," Gene Ther. 5, 1333 (1998).

${ }^{24}$ A. H. Jobe, T. Ueda, J. A. Whitsett, B. C. Trapnell, and M. Ikegami, "Surfactant enhances adenovirus-mediated gene expression in rabbit lungs," Gene Ther. 3, 775 (1996).

${ }^{25}$ O. E. Jensen, D. Halpern, and J. B. Grotberg, "Transport of a passive solute by surfactant-driven flows," Chem. Eng. Sci. 49, 1107 (1994).

${ }^{26}$ Y. L. Zhang, O. K. Matar, and R. V. Craster, "A theoretical study of chemical delivery within the lung using exogenous surfactant," Med. Eng. Phys. 25, 115 (2003).

${ }^{27}$ S. Iqbal, S. Ritson, I. Prince, J. Denyer, and M. L. Everard, "Drug delivery and adherence in young children," Pediatr. Pulmonol 37, 311 (2004).

${ }^{28}$ P. B. Myrdal, K. L. Karlage, S. W. Stein, B. A. Brown, and A. Haynes, "Optimized dose delivery of the peptide cyclosporine using hydrofluoroalkane-based metered dose inhalers," J. Pharm. Sci. 93, 1054 (2004).

${ }^{29}$ K. J. Cassidy, J. L. Bull, M. R. Glucksberg, C. A. Dawson, S. T. Haworth, R. B. Hirschl, N. Gavriely, and J. B. Grotberg, "A rat lung model of instilled liquid transport in the pulmonary airways," J. Appl. Physiol. 90, 1955 (2001).

${ }^{30} \mathrm{~S}$. L. Waters and J. B. Grotberg, "The propagation of a surfactant laden liquid plug in a capillary tube," Phys. Fluids 14, 471 (2002).

${ }^{31}$ P. D. Howell, S. L. Waters, and J. B. Grotberg, "The propagation of a liquid bolus along a liquid-lined flexible tube," J. Fluid Mech. 406, 309 (2000).

${ }^{32}$ H. Fujioka and J. B. Grotberg, "Steady propagation of a liquid plug in a 2-dimensional channel," ASME J. Biomech. Eng. 126, 567 (2004).

${ }^{33}$ G. M. Ginley and C. J. Radke, "Influence of soluble surfactants on the flow of long bubbles through a cylindrical capillary," ACS Symp. Ser. 396, 480 (1989).

${ }^{34}$ J. Ratulowski and H.-C. Chang, "Marangoni effects of trace impurities on the motion of long gas bubbles in capillaries," J. Fluid Mech. 210, 303 (1990).

${ }^{35}$ F. Wassmuth, W. G. Laidlaw, and D. A. Coombe, "Calculation of interfacial flows and surfactant redistribution as a gas-liquid interface moves between 2 parallel plates," Phys. Fluids A 5, 1533 (1993).

${ }^{36} \mathrm{~S}$. N. Ghadiali and D. P. Gaver, "The influence of non-equilibrium surfactant dynamics on the flow of a semi-infinite bubble in a rigid cylindrical capillary tube," J. Fluid Mech. 478, 165 (2003).

${ }^{37}$ M. Severino, M. D. Giavedoni, and F. A. Saita, "A gas phase displacing a liquid with soluble surfactants out of a small conduit: The plane case," Phys. Fluids 15, 2961 (2003).

${ }^{38}$ M. A. Krueger and D. P. Gaver, "A theoretical model of pulmonary surfactant multilayer collapse under oscillating area conditions," J. Colloid Interface Sci. 229, 353 (2000).

${ }^{39}$ D. R. Otis, Jr., E. P. Ingenito, R. D. Kamm, and M. Johnson, "Dynamic surface tension of surfactant TA: Experiments and theory," J. Appl. Physiol. 77, 2681 (1994).

${ }^{40} \mathrm{C}$. H. Chang and E. I. Franses, "Adsorption dynamics of surfactants at the air/water interface-A critical-review of mathematical-models, data, and mechanisms," Colloids Surf., A 100, 1 (1995).

${ }^{41}$ S. V. Patankar, Numerical Heat Transfer and Fluid Flow (Hemisphere, London, 1980).

${ }^{42}$ J. F. Thompson and Z. U. Warsi, "Boundary-fitted coordinate system for numerical solution of partial differential equations-A review," J. Comput. Phys. 47, 1 (1982).

${ }^{43}$ S. Muzaferija and M. Peric, "Computation of free-surface flows using the finite-volume method and moving grids," Numer. Heat Transfer, Part B 32, 369 (1997).

${ }^{44}$ J. F. Thompson, B. K. Soni, and N. P. Weatherill, Handbook of Grid Generation (CRC, Boca Raton, FL, 1999), p. 4.

${ }^{45}$ P. Wesseling, Principles of Computational Fluid Dynamics (Springer, New York, 2000), p. 538

${ }^{46}$ M. D. Giavedoni and F. A. Saita, "The axisymmetric and plane cases of a gas phase steadily displacing a Newtonian liquid-A simultaneous solution of the governing equations," Phys. Fluids 9, 2420 (1997).

${ }^{47}$ D. Halpern and D. P. Gaver, "Boundary-element analysis of the timedependent motion of a semiinfinite bubble in a channel," J. Comput. Phys. 115, 366 (1994)

${ }^{48}$ F. P. Bretherton, "The motion of long bubbles in tubes," J. Fluid Mech. 10, 166 (1961).

${ }^{49}$ M. Heil, "Finite Reynolds number effects in the Bretherton problem," Phys. Fluids 13, 2517 (2001).

${ }^{50}$ K. J. Cassidy, N. Gavriely, and J. B. Grotberg, "Liquid plug flow in straight and bifurcating tubes," ASME J. Biomech. Eng. 123, 580 (2001).

${ }^{51}$ M. A. Launoissurpas, T. Ivanova, I. Panaiotov, J. E. Proust, F. Puisieux, and G. Georgiev, "Behavior of pure and mixed Dppc liposomes spread or adsorbed at the air-water-interface," Colloid Polym. Sci. 270, 901 (1992).

${ }^{52} \mathrm{~S}$. N. Ghadiali and D. P. Gaver, "An investigation of pulmonary surfactant physicochemical behavior under airway reopening conditions," J. Appl. Physiol. 88, 493 (2000).

${ }^{53}$ S. Schurch, H. Bachofen, J. Goerke, and F. Possmayer, "A captive bubble method reproduces the in situ behavior of lung surfactant monolayers," J. Appl. Physiol. 67, 2389 (1989).

${ }^{54}$ M. L. Agrawal and R. D. Neuman, "Surface-diffusion in monomolecular films. 2. Experiment and theory," J. Colloid Interface Sci. 121, 366 (1988).

${ }^{55}$ W. R. Schief, M. Antia, B. M. Discher, S. B. Hall, and V. Vogel, "Liquidcrystalline collapse of pulmonary surfactant monolayers," Biophys. J. 84, 3792 (2003).

${ }^{56}$ M. C. Phillips and D. Chapman, "Monolayer characteristics of saturated I,2-diacyl phosphatidylcholines (lecithins) and phosphatidylethanolamines at air-water interface," Biochim. Biophys. Acta 163, 301 (1968).

${ }^{57}$ M. M. Lipp, K. Y. C. Lee, D. Y. Takamoto, J. A. Zasadzinski, and A. J. Waring, "Coexistence of buckled and flat monolayers," Phys. Rev. Lett. 81, 1650 (1998)

${ }^{58}$ H. E. Ries and H. Swift, "Twisted double-layer ribbons and the mechanism for monolayer collapse," Langmuir 3, 853 (1987).

${ }^{59}$ H. E. Ries, "Stable ridges in a collapsing monolayer," Nature (London) 281, 287 (1979).

${ }^{60}$ J. N. Hildebran, J. Goerke, and J. A. Clements, "Pulmonary surface-film stability and composition," J. Appl. Physiol.: Respir., Environ. Exercise Physiol. 47, 604 (1979).

${ }^{61}$ R. J. King and J. A. Clements, "Surface-active materials from dog lung. 2. Composition and physiological correlations," Am. J. Physiol. 223, 715 
(1972).

${ }^{62}$ C.-W. Park, "In fluence of soluble surfactants on the motion of finite bubble in a capillary tube," Phys. Fluids A 4, 2335 (1992).

${ }^{63}$ M. D. Giavedoni and F. A. Saita, "The rear meniscus of a long bubble steadily displacing a Newtonian liquid in a capillary tube," Phys. Fluids 11, 786 (1999).
${ }^{64}$ M. S. Borgas and J. B. Grotberg, "Monolayer flow on a thin film," J. Fluid Mech. 193, 151 (1988).

${ }^{65} \mathrm{~N}$. Antonova, R. Todorov, and D. Exerowa, "Rheological behavior and parameters of the in vitro model of lung surfactant systems: The role of the main phospholipid component," Biorheology 40, 531 (2003). 